\title{
Machine and Deep Learning Towards COVID-19 Diagnosis and Treatment: Survey, Challenges, and Future Directions
}

\author{
Tarik Alafif $^{\S}$, Abdul Muneeim Tehame ${ }^{\S}$, Saleh Bajaba, Ahmed Barnawi, and Saad Zia
}

\begin{abstract}
With many successful stories, machine learning (ML) and deep learning (DL) have been widely used in our everyday lives in a number of ways. They have also been instrumental in tackling the outbreak of Coronavirus (COVID-19), which has been happening around the world. The SARS-CoV-2 virus-induced COVID-19 epidemic has spread rapidly across the world, leading to international outbreaks. The COVID-19 fight to curb the spread of the disease involves most states, companies, and scientific research institutions. In this research, we look at the ML and DL based on Artificial Intelligence ( AI) for COVID19 diagnosis and treatment. Furthermore, in the battle against COVID-19, we summarise the AI-based ML and DL methods and available datasets, tools, and performance. This survey offers a detailed overview of the existing state-of-the-art methodologies and implementations for ML and DL researchers and the wider health community with descriptions of how ML and DL and data can improve the status of COVID-19, and more studies to avoid the outbreak of COVID-19. Details of challenges and future directions are also given.
\end{abstract}

Index Terms-COVID-19, diagnosis, treatment, artificial intelligence, machine learning, deep learning.

\section{INTRODUCTION}

$\mathbf{S}$ EVERE Acute Respiratory Syndrome Corona-Virus 2 (SARS-CoV-2) is currently a considerable infectious worldwide disease. Corona-virus 2019 (COVID-19) caused by a virus named SARS-CoV-2 was very first apprised in Wuhan, China in December 2019 and later in many parts all over the world. on January 03, 2020, the World Health Organization declared that COVID-19 is a Public Health Emergency of International Concern (PHEIC), and confirmed it as an epidemic on March 11, 2020. [84]. This disease has been accounted for in 216 countries and regions all around from May 16, 2020. The disease has spread and prompted momentous side effects with $31,668,942$ cases that confirmed coronavirus and 972,437 number of deaths on September 22, 2020.

T. Alafif is with the Department of Computer Science, Jamoum University College, Umm Al-Qura University, Jamoum, Makkah, 25375 Saudi Arabia e-mail: (see https://uqu.edu.sa/en/Profile/tkafif).

A. Tehame is with the Department of Software Engineering, Sir Syed University of Engineering and Technology, Karachi, 75300 Pakistan.

S. Bajaba is with Department of Business Administration, King Abdulaziz University, Jeddah, Makkah, 21589 Saudi Arabia.

A. Barnawi is with Faculty of Computing and Information Technology, King Abdulaziz University Jeddah, Makkah, 21589 Saudi Arabia.

S. Zia is with Jeddah Cable Company, Jeddah, Makkah, 31248 Saudi Arabia.

Manuscript received Month 00, 2020; revised Month 00, 2020.

$\S$ Equal contribution
The health industry is eagerly looking for new technologies and techniques to track and control the growth of coronavirus epidemic in this international health crisis. The one of the most uses global technology right now is Artificial Intelligence (AI) that can track the speed and detect the growth rate of the corona virus, identify the risk and severity of Corona virus pateints. AI can also anticipate the possibility of death by adequately analyzing previous patient data. Artificial intelligence can assist us with battling the virus by testing individuals, medical assistance, data and information, and recommendations about disease control.

In order to solve complex problems in our lives, AI is a broad umbrella consisting of many sub-areas. These subareas include learning, preparation, thinking, representation of information and searching. Machine Learning (ML) and Deep Learning (DL) are a subset of AI areas that consist of several algorithms that mimic human brains and behaviours based on data monitoring to identify or cluster particular tasks.

ML is a subset of AI, a traditional method of learning that needs only a small amount of knowledge to learn how to solve problems. Logistic Regression (LR), Decision Tree (DT), Random Forest (RF), K-nearest Neighbor (KNN), Adaboost, K-means clustering (KC), Density clustering (DC), Hidden Markov Models (HMM), Support vector machine (SVM), Naive Bayes (NB), and Multi-layer Neural Network (NN) or Artificial Neural Network (ANN) are ML techniques.

DL, on the other hand, is a subset of ML that focuses on building deep structural NN models that learn from data using algorithms of feedforward and backprobagation. After ML, the DL emerged and outperformed it in the last two decades in several activities. Nevertheless, it takes a huge amount of data to understand. Exceptional cases of DL where large-scale data is not needed to train have been transfer learning and generative models. DL algorithms usually involve Deep Belief Networks (DBN), Deep Neural Network (DNN), Convolutional Neural Networks (CNN), Restricted Boltzmann Machines (RBM), Recurrent Neural Networks (RNN) including Long-short-term-memory (LSTM), Autoencoder (AE), and Generative Adversarial Network (GAN).

Appreciatively, research works in industry, medical, technological, and military sectors has victoriously introduced advanced AI-based ML and DL methods in the COVID19 war with in short period after the outbreak of COVID19 and attained substantial progress. For example, across medical image analysis, ML and DL help COVID-19 diagnosis as well as provide non-invasive detection measure to 


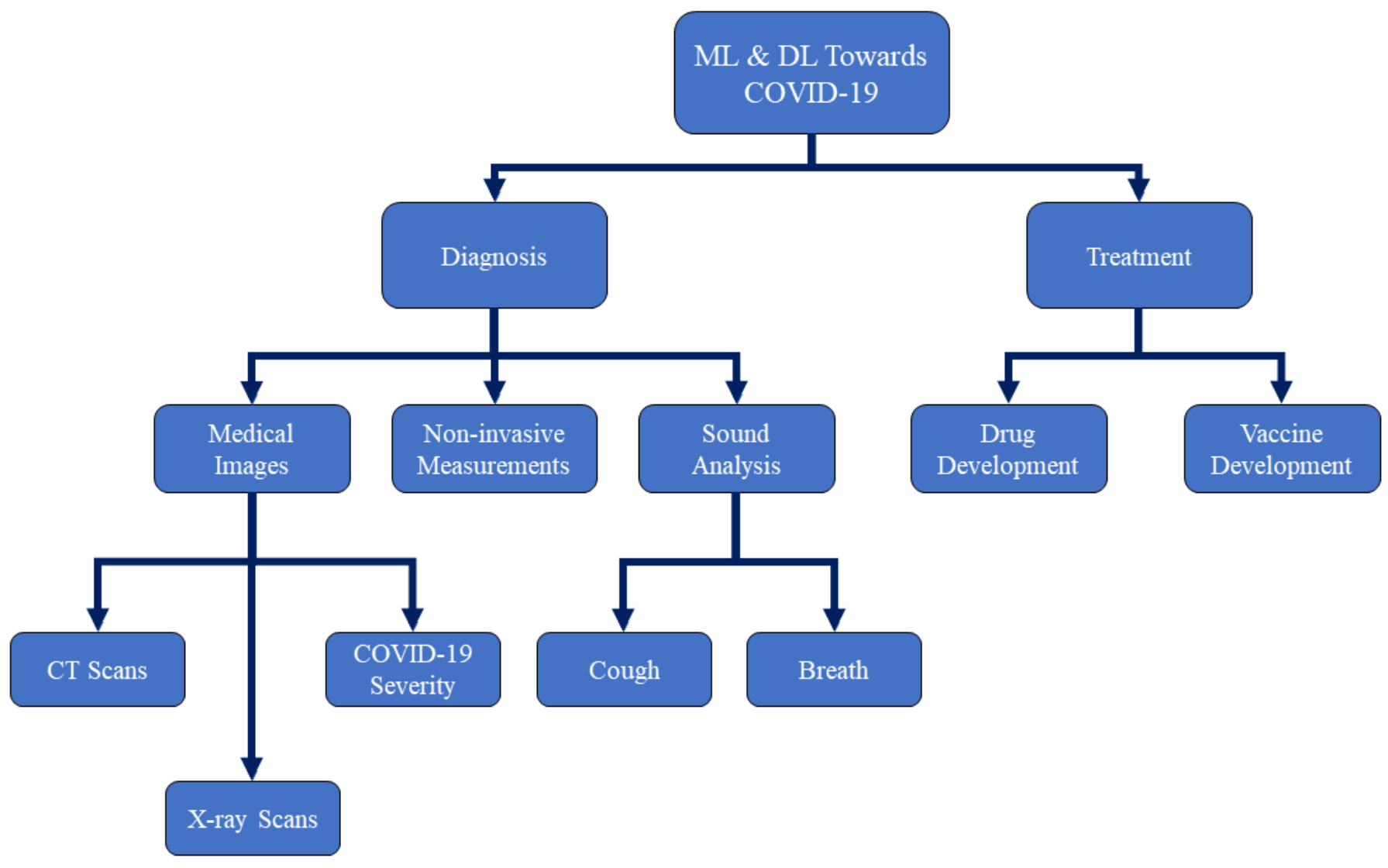

Fig. 1. A taxonomy of our survey on ML and DL research works towards COVID-19 diagnosis and treatment.

avoid medical personnel from contracting pathogens and for further treatment, the patient's severity score is also given. In virology studies, ML and DL are used to examine SARS$\mathrm{CoV}-2$ protein-related genetics and predict novel combinations that can be used for drug production and vaccination. In addition, on a large-scale, COVID-19 case data and social media data, AI intelligent models based on ML and DL learn to construct disease transmission models that accurately predict outbreaks, transmission path, transmission list, and effects. ML and DL are also vastly used in epidemic protection and public monitoring, such as security check-ups in airports, patient tracking, and epidemic detection.

In this survey, we introduce the main scope of AI focusing on ML and DL towards COVID-19 research incorporates the sides of disease diagnosis and drug and vaccine developments. Note that due to the fast evolution of the COVID-19 epidemic, we have quoted many published research works before a thorough investigation, where these works actually should be surveyed for their precision and quality in peer review. A taxonomy of our survey on ML and DL research works towards COVID-19 diagnosis and treatment is shown in Figure 1.

The remainder of this paper is organized as follows. In Section [I]. we provide details on the current AI-based ML and DL approaches for classifying COVID-19 from Chest $\mathrm{CT}$ and X-ray scans in addition to non-invasive COVID-19 measurements. We also show the COVID-19 severity detection using the DL-based methods. In Section III we provide details on the current AI-based ML and DL approaches for drug and vaccines development. In Section IV challenges and future work directions using AI-based ML and DL approaches for tackling the problem of COVID-19 are provided in details. Finally, our conclusion is provided in Section $\mathbf{V}$

\section{Medical Image Inception Using AI-Based ML AND DL FOR THE DETECTION OF COVID-19}

Nationally and internationally, the Coronavirus (COVID-19) outbreak is rising. In the universal battle against COVID-19, for example, medical imaging, X-ray and computed tomography (CT) play a key role, and the latest AI developments tend to improve the capacity of imaging tools and facilitate healthcare personnel.

Medical imaging research is commonly used for the identification of COVID-19 by clinicians. Chest X-ray and lung CT image samples are mostly used in COVID-19 clinical imaging trials. AI innovation plays a significant role in medical imaging testing. It has produced enormous results in image identification, organ recognition, geographic infection classification, and disease classification. It not only decreases the picture diagnostic time of the radiologist, but also increases the accuracy and execution of the diagnosis. Through correct diagnostic precision in X-ray and CT imaging, AI can enhance work performance, making it easier to test as follows. The 
TABLE I

A SUMmaRY OF HigheST PERFoRMANCE AI-BASED ML AND DL TECHNIQUES FOR DIAGNOSING COVID-19 USING CHEST X-RAY AND CT IMAGES.

\begin{tabular}{|c|c|c|c|c|c|}
\hline Author & Data & $\begin{array}{l}\text { ML/DL } \\
\text { Method }\end{array}$ & Accuracy/AUC & Sensitivity & Specificity \\
\hline Li et al. $\mid 45]$ & $\begin{array}{l}\text { a total of } 4,356 \text { CT images from } 3,322 \text { patients } \\
\text { obtained from six clinics ( } 1,296 \text { images for } \\
\text { COVID-19, 1,735 for CAP and } 1,325 \text { for non- } \\
\text { pneumonia). }\end{array}$ & $\begin{array}{l}\text { COVNet using } \\
\text { pre-traind } \\
\text { ResNet-50 }\end{array}$ & $96 \%$ AUC & $87 \%$ & $92 \%$ \\
\hline Butt et al. $[12]$ & $\begin{array}{l}\text { A total of } 618 \text { CT images ( } 219 \text { images from } 110 \\
\text { COVID-19 patients, } 224 \text { CT images from } 224 \\
\text { patients with influenza-A viral pneumonia, and } \\
175 \text { CT images from healthy people). }\end{array}$ & $\begin{array}{l}\text { Location- } \\
\text { attention } \\
\text { network and } \\
\text { ResNet-18 }\end{array}$ & $\begin{array}{l}86.7 \% \\
\text { Accuracy }\end{array}$ & N/A & N/A \\
\hline Ghoshal and Tucker [23] & $\begin{array}{l}\text { A total of } 5,941 \text { CT images for four classes } \\
(1,583 \text { normal, } 2,786 \text { bacterial pneumonia, } \\
1,504 \text { viral pneumonia, and } 68 \text { COVID-19) }\end{array}$ & $\begin{array}{l}\text { Drop-weights } \\
\text { based Bayesian } \\
\text { CNNs }\end{array}$ & $\begin{array}{l}89.92 \% \text { Accu- } \\
\text { racy }\end{array}$ & N/A & N/A \\
\hline Bai et al. $|6|$ & $\begin{array}{l}\text { Unknown number of chest CT images collected } \\
\text { from } 133 \text { COVID- } 19 \text { patients of which } 54 \text { pa- } \\
\text { tients had severe/critical cases. }\end{array}$ & $\begin{array}{l}\text { MLP } \quad \text { and } \\
\text { LSTM }\end{array}$ & $95 \%$ AUC & $95 \%$ & $95 \%$ \\
\hline Jin et al. $|39|$ & $\begin{array}{l}496 \text { chest CT scans from COVID-19 patients } \\
\text { and } 1,385 \text { non-COVID-19 CT scans. }\end{array}$ & $\mathrm{CNN}$ & $97.17 \%$ AUC & $90.19 \%$ & $95.76 \%$ \\
\hline Jin et al. $|40|$ & $\begin{array}{l}\text { A total of } 1,136 \text { CT images ( } 723 \text { COVID-19 } \\
\text { confirmed COVID-19 cases from five hospitals). }\end{array}$ & $\begin{array}{l}\text { 3D UNet++ and } \\
\text { ResNet-50 }\end{array}$ & N/A & $97 \%$ & $92 \%$ \\
\hline Narin et al. $|56|$ & $\begin{array}{l}\text { Only } 50 \text { COVID- } 19 \text { chest X-ray scans and } 50 \\
\text { for non-COVID- } 19 \text {. }\end{array}$ & $\begin{array}{l}\text { Pre-trained } \\
\text { ResNet-50 }\end{array}$ & $98 \%$ Accuracy & N/A & N/A \\
\hline Wang and Wong [93] & $\begin{array}{l}16,756 \text { chest X-ray scans from } 13,645 \text { COVID- } \\
19 \text { patients. }\end{array}$ & COVID-Net & $\begin{array}{l}92.4 \% \\
\text { Accuracy }\end{array}$ & $91.0 \%$ & N/A \\
\hline Gozes et al. [24] & $\begin{array}{l}\text { Unknown number of CT scans collected from } \\
157 \text { Chinese and U.S. COVID-19 patients. }\end{array}$ & ResNet-50 & $97 \%$ AUC & $98.2 \%$ & $92.2 \%$ \\
\hline Chowdhury et al. $\mid 15]$ & $\begin{array}{l}\text { A total of } 3,487 \text { chest CT scan images } \\
(1,579 \text { normal, } 1,485 \text { viral pneumonia, and } 423 \\
\text { COVID- } 19 \text {. }\end{array}$ & $\begin{array}{l}\text { AlexNet, } \\
\text { ResNet-18, } \\
\text { DenseNet201, } \\
\text { and SqueezeNet }\end{array}$ & $\begin{array}{l}99.7 \% \\
\text { Accuracy }\end{array}$ & $99.7 \%$ & $99.5 \%$ \\
\hline Maghdid et al. |47| & $\begin{array}{l}\text { A total of } 170 \text { COVID-19 X-ray scans and a } \\
\text { total of } 361 \text { COVID-19 CT scans of collected } \\
\text { from five different places. }\end{array}$ & $\begin{array}{l}\text { CNN and pre- } \\
\text { trained AlexNet } \\
\text { models }\end{array}$ & $\begin{array}{l}\text { Accuracy } \\
98 \% \text { of } \\
\text { ray scans } \\
94.1 \% \text { ond } \\
\text { scans }\end{array}$ & $\begin{array}{l}100 \% \\
\text { on X-ray } \\
\text { scans and } \\
90 \% \text { on } \\
\text { CT scans }\end{array}$ & N/A \\
\hline $\begin{array}{l}\text { Apostolopoulos and Mpe- } \\
\text { siana }[\overline{5}]\end{array}$ & $\begin{array}{l}\text { A total of } 1,428 \text { chest } X \text {-ray scans ( } 224 \text { COVID- } \\
19,700 \text { pneumonia patient, and } 504 \text { normal). }\end{array}$ & $\begin{array}{l}\text { Pre-trained } \\
\text { VGG-19 }\end{array}$ & $\begin{array}{l}93.48 \% \text { Accu- } \\
\text { racy }\end{array}$ & $92.85 \%$ & $98.75 \%$ \\
\hline Sethy and Behera $|79|$ & $\begin{array}{l}\text { Chest X-ray scans of } 25 \text { COVID- } 19 \text { positive and } \\
25 \text { negative. }\end{array}$ & $\begin{array}{l}\text { ResNet-50 and } \\
\text { SVM }\end{array}$ & $\begin{array}{l}93.28 \% \text { Accu- } \\
\text { racy }\end{array}$ & $97 \%$ & $93 \%$ \\
\hline Hemdan et al. [27] & $\begin{array}{l}\text { Chest X-ray scans } 25 \text { COVID-19 positive and } \\
25 \text { Normal patient. }\end{array}$ & COVIDX-Net & $90 \%$ Accuracy & N/A & N/A \\
\hline Wang et al. $\mid 94]$ & $\begin{array}{l}\text { Chest CT scans of } 195 \text { COVID-19 positive and } \\
258 \text { COVID-19 negative patients. }\end{array}$ & M-Inception & $\begin{array}{l}89.5 \% \\
\text { Accuracy }\end{array}$ & $87 \%$ & $88 \%$ \\
\hline Zheng et al. $|103|$ & $\begin{array}{l}\text { Chest CT images of } 313 \text { COVID-19 and } 229 \\
\text { non-COVID-19 patients. }\end{array}$ & $\begin{array}{l}\text { U-Net and a 3D } \\
\text { Deep Network }\end{array}$ & $90 \%$ Accuracy & $95 \%$ & $95 \%$ \\
\hline Hall et al. $|25|$ & $\begin{array}{l}\text { A total of } 455 \text { chest X-ray ( } 135 \text { of COVID-19 } \\
\text { and } 320 \text { of viral and bacterial pneumonia. }\end{array}$ & $\begin{array}{l}\text { Pre-trained } \\
\text { ResNet-50 }\end{array}$ & $\begin{array}{l}89.2 \% \\
\text { Accuracy } \\
\text { and } 95 \% \text { AUC }\end{array}$ & N/A & N/A \\
\hline Ni et al. $|57|$ & $\begin{array}{l}\text { CT images of } 96 \text { COVID- } 19 \text { positive collected } \\
\text { from three hospitals in China. }\end{array}$ & Deep CNN & $73 \%$ Accuracy & $95 \%$ & $95 \%$ \\
\hline Apostolopoulos et al. [4] & $\begin{array}{l}\text { A total of 3,905 X-ray scans for several classes } \\
\text { of disease including COVID- } 19 .\end{array}$ & MobileNet-v2 & $\begin{array}{l}99.18 \% \text { Accu- } \\
\text { racy }\end{array}$ & $97.36 \%$ & $99.42 \%$ \\
\hline Shi et al. $[81]$ & $\begin{array}{l}\text { CT images of } 2,685 \text { collected from three hos- } \\
\text { pitals, } 1,658 \text { of COVID- } 19 \text { positive cases and } \\
1,027 \text { cases were CAP patients. }\end{array}$ & $\mathrm{RF}$ & $\begin{array}{l}87.9 \% \\
\text { Accuracy }\end{array}$ & $90.7 \%$ & $83.3 \%$ \\
\hline Loey et al. $\mid 46]$ & $\begin{array}{l}\text { Chest X-ray scans of } 307 \text { for COVID-19, } \\
\text { normal, pneumonia, bacterial, and pneumonia } \\
\text { virus. }\end{array}$ & $\begin{array}{l}\text { GAN with } \\
\text { AlexNet, } \\
\text { GoogLeNet, } \\
\text { and ResNet18 } \\
\text { pre-trained } \\
\text { models. }\end{array}$ & $\begin{array}{l}100 \% \text { Accuracy } \\
\text { for two classes, } \\
85.19 \% \text { for } \\
\text { three classes, } \\
\text { and } 80.56 \% \text { for } \\
\text { the four classes }\end{array}$ & N/A & N/A \\
\hline
\end{tabular}


computer-aided networks also assist radiologists in making clinical decisions, i.e. for the identification , monitoring, and prognosis of diseases. We will address the innovations of AI techniques to chest X-ray and CT imaging in depth.

1) Chest CT Image Detection: A valued feature of the assessment of patients with doubtful SARS-CoV-2 infection is the chest CT picture. There is a growing research on the role of COVID-19 imaging for treatment and diagnosis. The infection triggers a huge spectrum of CT scan imaging discoveries, most commonly ground-glass opacities and lung periphery consolidations. Chest CT sensitivity to diagnose COVID-19 has been found to be significantly higher and can occur prior to a positive viral lab test. Therefore, hospitals with a large quantities of admissions use CT for the fast emergency of patients with conceivable COVID-19 disease in epidemic territories where the basic healthcare system is under pressure. In the estimation of COVID-19 patients with severe and compound respiratory symptoms, chest CT plays a vital role. Based on scans, it is possible to determine how badly the lungs are compromised and how the illness of the individual progresses, which is effective in making medical decisions.There is a growing understanding of the sudden incidence of lung defects induced by COVID-19 in CT scans conducted for many other clinical indications, such as abdominal CT scans for bowel disorders or patients without respiratory symptoms [58]. In this pandemic, by reducing the strain on clinicians, the evaluation of AI may become the most significant factor. Although it can take up to 15 minutes to manually interpret a CT scan, AI can analyse the images in 10 seconds. [58]. Therefore, advanced image processing with artificial neural network has the possibility to significantly improve the function of CT in COVID-19 detection by allowing a large proportion of patients to identify disease easily and rapidly with accuracy. The continuation of AI-based CT imaging tests usually involves the following steps: regional division of the Region of Interest (ROI), removal of pulmonary tissue, identification of regional infection, and classification of COVID-19. A basic basis for analysing AI-based imagery is the recognition of lung organs and ROIs. Demonstrates ROI for further testing and analysis in CT imaging in lungs, lung lobes, bronchopulmonary segments, and regions with infection or ulcers). For CT image classification, different types of DL networks , e.g. U-Net, V-Net, and VB-Net, VNET-IRRPN, had been used. Of a overall of 905 patients assessed with real-time RT-PCR assay and next-generation RT-PCR, 419 , about $(46.3 \%)$, were confirmed by an AI device with SARS-CoV-2. The AI method consists of deep CNN for the primary CT scan to evaluate the image characteristics and attributes of individuals with SARS-CoV-2. Then, according to clinical knowledge, SVM, RF, and MLP classifiers were used to identify SARS-CoV-2 patients. To foresee COVID19 status, the AI system operates on radiological data and medical factors. The deep CNN-based AI system obtained an AUC of $92 \%$ and had a comparable sensitivity relative to the senior thoracic radiologist in the experimental set of 279 patients. Furthermore, the Artificial Intelligence (AI) system enhanced the detection of patients who aimed for RT-PCR detected COVID-19 who submitted standard CT scans, which correctly classified 17 out of 25 patients (68\%). and all of these patients were graded by radiologists as COVID-19 negative [50]. The training dataset also included 25 COVID-19 positive cases with a chest CT marked as negative by the two reading radiologists during display. The CNN-based model categorized 13 out of 25 , about $(52 \%)$ of images, as positive for COVID-19. The clinical model categorized 16 out of 25 , about $(64 \%)$ of images, as positive for disease, and the joint model categorized 17 out of $25(68 \%)$ as positive for disease, while the senior radiologist and their fellows classified 0 out of $25(0 \%)$ of these images as positive for disease [50] .

In attempt to discover the answer that could rapidly decompose images from deep learning and recognize COVID-19 features, researchers are trying to develop several AI resources. Research team led by Bo Xu of the Tianjin Medical University Cancer Institute and Hospital involved CT scans of 180 patients confirmed to have severe viral pneumonia even before epidemic of COVID-19 and 79 patients with certified COVID19 to establish an AI method to classify COVID-19 [65]. They gave pictures of patients at random to train and test a deep CNN-based algorithm. In the findings released in medRxiv [94], The researchers stated that their model detected COVID19 with $89.5 \%$ accuracy from CT images. An accuracy of about $55 \%$ was reported by two radiologists who analysed the images. The team confirms the findings indicate that from a CT scan, AI can provide an exact analysis. Another algorithm named as RADLogics algorithm [38] that managed to detect and assist in initiating a COVID-19 patient's improvement.

Two studies, published in [50] and [101], advance this thought by utilizing DL trained on CT scans as a fast symptomatic instrument to search for COVID-19 infection in sufferers who were admitted to the hospitals and required medical image processing. In [101], researchers at Macau University of Science and Technology applied 532,000 CT images from 3,777 patients in China to train and test their AIbased models, concentrating on the tell-tale lesions observed in COVID-19 patient lungs. The AI model successfully diagnosed coronavirus-induced pneumonia no less $85 \%$ of the time when it was used in a database of 417 patients in four separate groups in a pilot study across many Chinese hospitals.

There seems to be a big problem in distinguishing whether the signs are COVID-19 and pneumonia detected in CT images by radiologists. A company, VIDA Diagnostics [92], has developed a LungPrint device that utilizes AI to analyse CT scans to accurately identify respiratory disorders, including COVID-19 signs and symptoms. In [2] NIH and NVIDIA researchers attempted to create a DL technique to detect COVID-19 using chest CT images utilizing datasets from four hospitals across China, Italy and Japan. In total, in this study, researchers used 2,724 samples from 2,619 patients, including two models (i.e. Full 3D, Hybrid 3D) used in series to establish the final prediction model for COVID-19. These two model work. The first model implemented a fixed input size (full 3D) of the entire lung area. At fixed image resolutions (hybrid $3 \mathrm{D})$, the second model used an average score for a few regions in each lung. The hybrid 3D model achieved $92.4 \%$ validation accuracy when detecting COVID-19 as well as other pneumonia, whereas the full 3D model achieved $91.7 \%$ 
accuracy.

To retrieve ROIs from each CT image and to acquire a training curve for suspected lesions, Chen et al. [14], constructed a U-Net++ deep learning structure. 46,096 anonymous images were collected and processed for model creation and validation from 106 patients already admitted, including 51 laboratory patients who had reported COVID-19 pneumonia and 55 other disease control patients at Renmin Hospital of Wuhan University in China. On February 5, 2020, twentyseven consecutive patients experiencing CT scanning were grouped at Renmin Hospital of Wuhan University to estimate the effectiveness of radiologists compared to the 2019-CoV pneumonia model. The U-Net++ model achieved overall of sensitivity of $100 \%$, specificity of $93.55 \%$, accuracy of $95.24 \%$ using retrospective dataset. The AI-based InferReadTM CT pneumonia method was used by Huang et al. [35], to accurately assess improvements in the lung burden of COVID19 patients. Three modules are incorporated into the tool: pulmonary and lobe extraction, classification of pneumonia, and measurable analysis. The CT image characteristics for COVID-19 pneumonia are divided into four modalities: mild , moderate, severe, and critical. A professional deep learning software automatically calculated the level of CT lung natural action of the overall lung and five lobes and compared CT scans over follow-up. A total of 126 COVID-19 patients including 6 mild, 94 moderate, 20 severe and 6 critical cases, were assessed. The rate of CT-based natural action was entirely diverse among the initial clinical groups, progressing gradually from mild to severe (all $\mathrm{P}<1 \%$ ).

71 CT scans from 52 COVID-19 approved patients in 5 hospitals were obtained by Qi et al. [67]. To excerpt 1,218 traits from each CT image, they used the Pyradiomics methodology. Models of CT radiomics focused on LR and RF algorithims. They were built on pneumonia lesion extracts during training and interactions. At the lung lobe and at the patient level, predictability efficacy was also evaluated in the experimental database. Types of CT Radiomics are focused on 6 second order. They were successful in distinguishing for short-term and long-term stays in patients with SARS-CoV2-related pneumonia, with 97\% AUC and 92\% LR and RF, respectively. The LR model showed $100 \%$ and $89 \%$ sensitivity and specificity while the RF model showed $75 \%$ and $100 \%$ similar sensitivity and specificity. The short-term hospital stay is less than 10 days, while the long-term hospital stay is more than 10 days.

2) Chest X-Ray Image Detection: As a highly helpful method for evaluating and testing COVID-19 patients, chest Xrays have been proposed. Figure 3 shows representative architectures of DL-based CT image classification and COVID-19 examination. Compared with CT images, chest X-ray (CXR) images are simpler to acquire in clinical radiology examinations. There are many available study [63] [7] that operates on chest X-ray (CXR) images for corona virus detection. For the most part, the CXR image testing factor based on AI strategies involves measures such as data correction, model training, and segmentation of COVID-19. There are several methodologies of deep learning (such as CNN, nCOVnet, U$\mathrm{Net}++)$ that are used to find better and fast detection in the detection of COVID-19 on X-ray images.

In medical centers and hospitals, X-ray devices deliver less costly and quicker outcomes from scanning different human organs. Interpretation of numerous X-ray images is typically performed by radiologists manually. Radiologists are only equipped to detect $69 \%$ of X-ray COVID-19 cases [8]. Pretrained models made it much easier and quicker to detect COVID-19. In [63], a dataset of reported positive COVID-19, typical bacterial pneumonia, and stable (no infection) cases was used. In this survey, a total of 1,428 X-ray scans were applied. The authors used the pre-trained model of VGG-16 to model the role of division and perform the categorization. In two and three production class cases, the examiner obtained $96 \%$ and $92.5 \%$ accuracy, respectively. Based on the results obtained, X-ray images can be accessed by the medical community as a possible symptomatic method for immediate and faster COVID-19 identification to supplement current diagnostic and symptomatic approaches. For better results by CXR images towards the war with SARS-nCOV2, several more creative algorithms are used. By assessing essential characteristics from chest X-ray scans, Basu and Mitra [7] introduced a domain transfer learning for detecting COVID-19. The definition of Gradient Class Activation Map (Grad-CAM) is also used on the collection of 20,000 Chest Xrays to get an account of the COVID-19 detection. To obtain an overview of the viability of using chest X-Rays tuned for classification between four groups, i.e. normal, other disease, pneumonia and COVID19 data were used to diagnose the disease using a 5-fold cross validation. With $100 \%$ of the COVID-19 and normal cases being accurately characterised in each validation fold, the overall accuracy was computed as 95.3\%. A misdiagnosis in both pneumonia and other disease stages has occurred.

Students Were also involved in the production of ML algorithm to identify novel COVID-19 patients. With the assistance of AI, Cranfield University students developed computer models that can diagnose COVID-19 in CXR images. [88] [89]. ML and DL techniques were used in the proposed models to obtain characteristics and identify CXR images. It can discern data that would not generally be apparent to the naked human eye and aid with COVID-19 detection.

The first model is to examine abnormalities in an X-ray, distinguishing patients with normal and pneumonia. The second model further operates on certain patients with pneumonia to determine if the COVID-19 virus causes pneumonia. [88] [89]. Figure 3 shows a high-level representation of the intelligent computational models developed at Cranfield University.

In the meantime, previous researches at King's College London, Massachusetts General Hospital and health tech company Zoe have begun studies of a fully AI-based identification that aims to predict COVID-19 pathogens by means of assessing the symptoms with the implications of traditional COVID19 tests [87]. With the support of Chest X-ray scans for COVID-19 diagnosis, another model [61] was proposed to provide an end-to-end structure without even using extracting features tool. This model is built on the foundations of Darknet-19 (form based on a real-time object detection method called YOLO) named DarkCovidnet, [61] which is already 


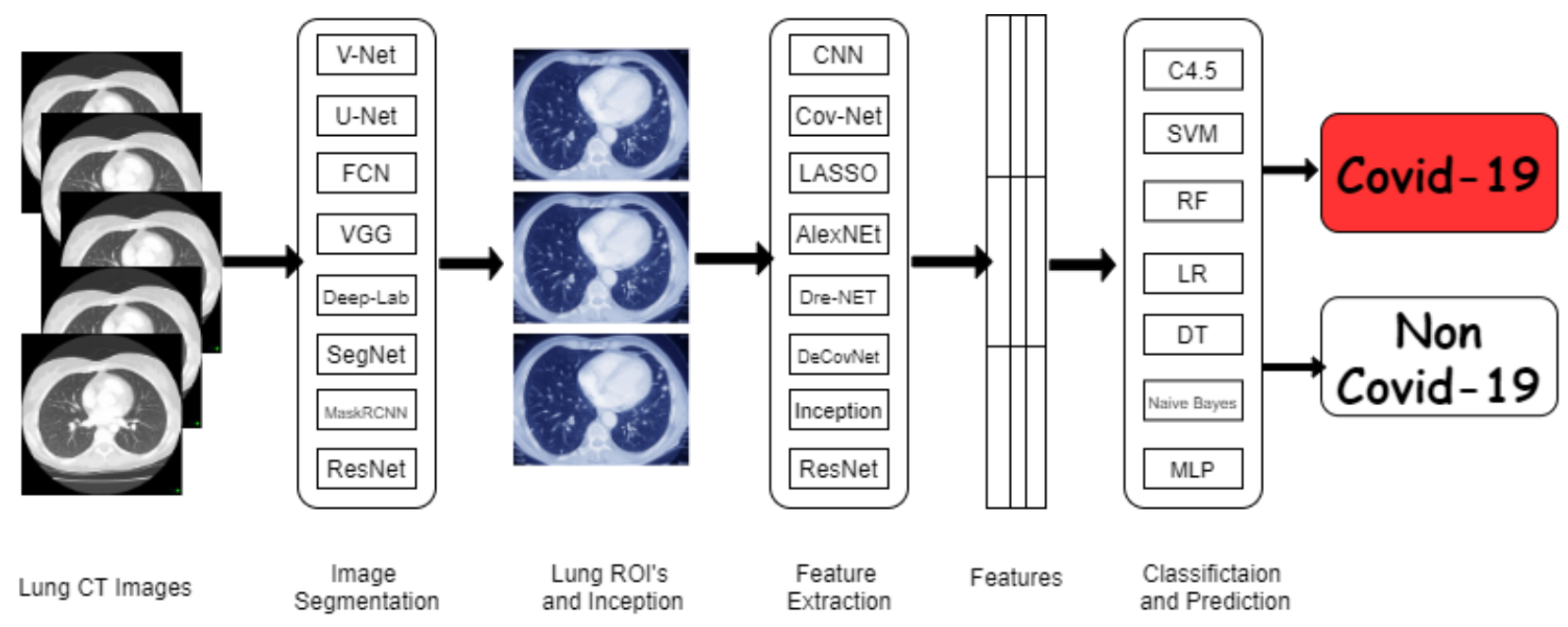

Fig. 2. Representative architectures of DL-based CT scan COVID-19 detection [13].

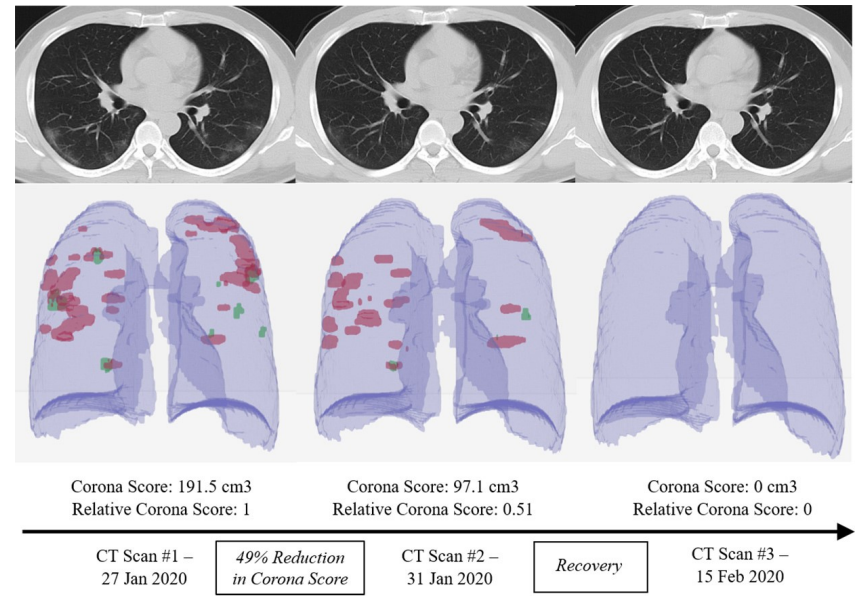

Fig. 3. Using different CT scans from a a COVID-19 patient, the RADLogics algorithm measures the recovery amount using a specific score [77].

documented. The achieved $85.35 \%, 92.18 \%$, and $87.37 \%$ of sensitivity, specificity, and F1-score values, respectively using the same model.

Most research studies are conducted using various methods to resolve COVID-19. A COVID-19 AI-based Detector (CovidAID) was proposed by Mangal et al. [49] CovidAID is a deep neural network model developed on the publicly available covid-chestxray-dataset dataset to treat patients for proper examination. With $100 \%$ sensitivity, they achieved $90.5 \%$ accuracy. To demonstrate COVID-19 positive X-rays from other negative ones, a deep learning-based CNN model, called Truncated Inception Net, was proposed in [17], six distinctive dataset forms were carried out by means of the types of $\mathrm{X}$ rays: COVID-19 positive, pneumonia positive, tuberculosis positive and normal cases. In detecting COVID-19 positive cases from total Pneumonia and healthy patients, their model obtained $99.96 \%$ of accuracy (AUC of 100\%). Comparably, in classifying COVID-19 positive patients from other types of X-ray scans, it obtained an accuracy of $99.92 \%$ (AUC of $99 \%$ ). They proved the feasibility of employing the proposed
Truncated Inception Net as a screening method, according to [17], and outperformed all existing tools. A research on COVID-19 prediction in X-ray images using transfer learning was published by Minaee et al [51], Four common pretrained deep convolution neural networks (CNNs), which are ResNet18, ResNet50, SqueezeNet, and DenseNet-161, were compared with their predictions They use COVID-19 and non-COVID datasets to instruct these 4 models, including 14 subclasses containing normal ChexPert dataset images. The models resulted an average specificity rate of $\sim 90 \%$ with a sensitivity range of $97.5 \%$. This highly supports the possibility that CXR imaging will distinguish COVID-19 from other infections and normal lung conditions. A COVID-19 assessment DT classifier from Chest X-ray Imaging is applied by [99]. It included three binary DTs, each of which was trained by a deep learning model built on the PyTorch system with a CNN. The Chest X-Ray images were graded as normal or abnormal by the first DT. The second tree differentiated the abnormal X-ray images containing tuberculosis symptoms, while the third tree distinguished the equivalent second for COVID-19. The accuracy of the first DT is $98 \%$ and the second DT is $80 \%$, while the third DT's average accuracy is $95 \%$. They claimed that the suggested deep learning-based DT classifier can be applied before RT-PCR outcomes are available in pre-screening instances to perform triage and fast-track conclusion making. GAN (Generative Adversarial Network) was introduced in a study [46] with deep transfer learning for COVID-19 identification in chest X-ray scans with a shortage of chest x-ray images dataset. 307 images for 4 different class groups, i.e. COVID-19, normal, bacterial pneumonia, and virus pneumonia were collected. Three AlexNet, GoogLeNet, and ResNet18 deep transfer models were chosen for operation. In this analysis, three case scenarios were tested: the first scenario consists of four dataset classes, while the second scenario consists of three classes, and the third scenario consists of only two classes. It was must to have the COVID-19 class in all scenarios. In the first scenario, as it attained $80.6 \%$ in testing accuracy, GoogLeNet was chosen to be the essential deep transfer model. With the use of Alexnet, the second 
scenario obtained a testing accuracy of $85.62 \%$. GoogLeNet was selected as a fundamental deep transfer learning model in the third scenario (which involves two classes, i.e. COVID-19 and normal), which gave an ideal $100 \%$ accuracy in testing and $99.9 \%$ accuracy in validation.

Especially in the low-resource X-ray settings may play a significant function in COVID-19 triage. 24,678 X-ray images were used to classify COVID-19 [68]. A deep learningbased AI framework CAD4COVID-Xray was trained. A lung segmentation with U-net and a CNN was implemented. 454 images were analyzed from a dataset (223 patients tested positive for COVID-19, the remaining 231 tested negative for COVID-19). Chest X-ray images were specifically labelled as COVID-19 pneumonia with an AUC of $81 \%$ by the AI system CAD4COVID-XRay. According to author [68] as part of a process of looking at symptomatic issues, the system can be useful, especially in low-resource settings where diagnostic equipment is not accessible. The Table I provides a summary of the best score AI-based ML and DL methods for diagnosing COVID-19 using radiology images.

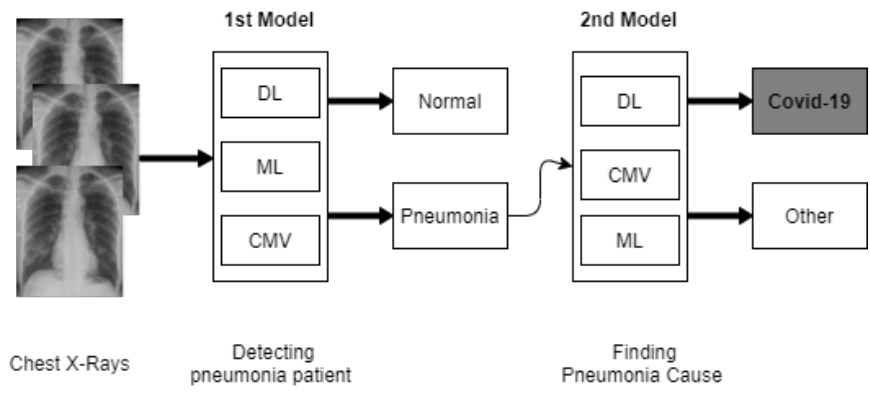

Fig. 4. Our representation of the proposed computation intelligent models used at Cranfield University.

3) COVID-19 Severity Classification Using Chest X-ray With Deep Learning: With the support of artificial intelligence, chest X-rays enable us to understood more, particularly by using ML and DL techniques. Chest X-rays (CXRs) offer a non-invasive method for monitoring disease progression. For front-chest X-ray pictures, a severity score predictor model of COVID-19 pneumonia is being studied in [16]. Expansions of lung involvement and light intensity are also included in the CXR image database. A pre-trained neural network model (i.e. DenseNet) in large chest X-ray sets (non-COVID-19) is used to create features of COVID-19 images to predict this activity. 94 images of COVID-19 certified patients go to the study the severity of COVID-19 prediction using DL as shown in Figure 5. A score-based methodology is used which includes two forms of scores. Table II provides examples of disease severity stages: the level of lung participation and the degree of ambiguity shown at a time in a single X-ray image.

For each lung, the level of lung involvement by ground glass ambiguity and degree of unification were rated. The overall degree and ambiguity score on both right and left lungs ranged from 0 to 8 and 0 to 6 , respectively. The severity of a disease that are used for increase or decrease of care, as well as tracking the effectiveness of patient treatment, especially in the ICU, can be found on the basis of the score.
TABLE II

COVID-19 SEVERITY STAGES USING A SCORE-BASED SYSTEM.

\begin{tabular}{|l|l|l|l|l|l|}
\hline Parameters & \multicolumn{5}{|c|}{ Severity Score } \\
\cline { 2 - 6 } & $\mathbf{0}$ & $\mathbf{1}$ & $\mathbf{2}$ & $\mathbf{3}$ & $\mathbf{4}$ \\
\hline $\begin{array}{l}\text { Extent of } \\
\text { Lung In- } \\
\text { volvement }\end{array}$ & No & $<25 \%$ & $25 \%-50 \%$ & $\begin{array}{l}50 \%- \\
75 \%\end{array}$ & $>75 \%$ \\
\hline $\begin{array}{l}\text { Degree of } \\
\text { ambiguity }\end{array}$ & No & $\begin{array}{l}\text { Ground } \\
\text { glass } \\
\text { ambiguity }\end{array}$ & unification & $\begin{array}{l}\text { White- } \\
\text { out }\end{array}$ & - \\
\hline
\end{tabular}

Mobile app development utilises AI to evaluate the severity of COVID-19 in the game as well. In [20] [91], a mobile app was created by researchers from NYU College of Dentistry that used a dataset of 160 images of reported patients with COVID-19 from China. Using the mobile app, the numerous bio-markers contained in the blood will diagnose COVID-19 severity levels from level 0 (mild) to 100 (extreme).

Ridley [73] on the other hand, developed a special form of deep-learning algorithm called the Convolutionary Siamese Neural Network (CSNN) to generate a score of COVID-19 patient pulmonary X-ray severity (PXS) and related well with radiologist evaluations and could also help predict whether a patient will require intubation or not before dying. The algorithm was carried out with two kinds of internal and external datasets. Internal research was conducted on a dataset of 154 COVID-19 admission chest X-rays, of which 92 had additional chest X-ray follow-up and were used for longitudinal study. In a community hospital - Newton-Wellesley Hospital in Newton, MA in the United States, external testing was performed on 113 consecutive admission chest X-rays from COVID-19 cases. Researchers found that the median PXS score was higher in intubated or deceased patients (PXS score $=7.9$ ) compared to those who had no intubation (PXS score $=3.2)$ on all test sets. The difference $(\mathrm{p}<0.001)$ was statistically important.

Qi et al. [67], obtained 71 CT scans from 52 COVID-19 patients registered in five hospitals. They applied the methodology of pyradiomics to extract 1,218 attributes from all CT images. Logistic regression (LR) and random forest (RF) based CT radiomics models were built on pneumonia lesion extracts in testing and interactions. At the lung lobe and at the patient level, predictability efficiency was also evaluated in the experimental database. Types of CT in radiology are based on 6 second order that have been functional in distinguishing short-term and long-term retention in patients with SARSCoV-2-related pneumonia, with $97 \%$ and $92 \%$ AUC by LR and $\mathrm{RF}$ respectively in the testing phase. The LR model resulted $100 \%$ of sensitivity and $89 \%$ of specificity while the RF model resulted $75 \%$ and $100 \%$ in sensitivity and specificity in the test results. Hospital stay for short-term is less or equal than 10 days while hospital stay for long-term is larger than 10 days.

4) Observing COVID-19 Through AI-based Cough Sound Analysis: Coughing is a symptom of more than 30 medical conditions that are not COVID-19. This makes COVID-19 infection identification by coughing alone a big challenge for different problems. Physicians also use sound signals made by human bodies. Examples of these sounds are moaning, breath- 

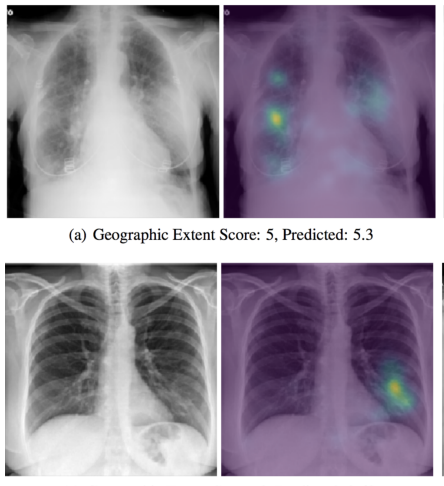

(c) Geographic Extent Score: 2, Predicted: 0.62 (a) Geographic Extent Score: 5, Predicted: 5.3

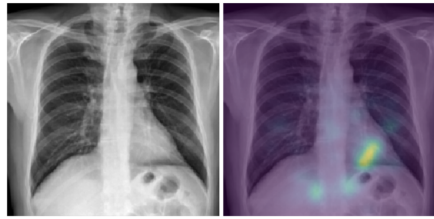

(b) Geographic Extent Score: 0 , Predicted: -0.8

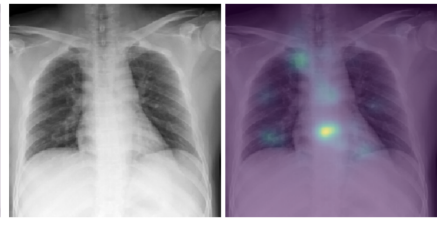

(d) Geographic Extent Score: 0, Predicted: 1.05
Fig. 5. A presentation of a predicted severity scores for COVID-19 chest X-ray scans using DenseNet model [16].

ing, heartbeat, digestion, vibrating sounds. They ar considered as indicators of diagnosis. Up to this point, at scheduled visits, such signals were usually collected via manual auscultation. Other research is being conducted for cardiovascular and respiratory studies using digital technologies to capture body sounds (e.g. from digital stethoscopes), which can be used for automatic COVID-19 analysis. Recent research has begun to investigate how respiratory sounds (e.g., cough, breathing, and voice) recorded in hospital by devices from patients tested positive for COVID-19 differ from healthy people's sounds.

A analysis of identification of COVID-19 based on coughs collected via phone app is reported in [36], using a cohort of 48 COVID-19, 102 bronchitis, 131 pertussis and 76 normal cough sounds to learn and evaluate this diagnostic method. Equation [1] is used in [36] to transform the collected cough data into Mel scale $m$ for data pre-processing:

$$
m=2595 \times \log _{10}\left(1+\frac{f}{700}\right)
$$

Another mobile app named kAs was built in [30], by Zensark Technologies (Hyderabad, Telangana, India) using machine learning to assess patient respiratory health and diseasespecific cough signatures. The app provides 15 questions about the subject and their cough sound into the mobile app to transmit the cough sounds to Swaasa, the company's AI platform, where on the basis of the answers to the questionnaire and the coughing tone, it goes to an audiometric examination and generates a COVID-19 Risk Score. A rating on a scale of 1 to 10 is produced by the app, where 10 is the highest risk level. It may take days or weeks to monitor person's risk score.

The effective use of Computer Audition (CA) and AI in the study of cough sounds in COVID-19 patients was explored by Schuller et al. [76] They first tested the capacity of CA to detect speech and cough automatically in different conditions such as breathing, dry and wet cough or sneezing, flu-like speech, eating habits, sleepiness, or pain. They subsequently recommended using the CA technology in the diagnosis and cure for COVID-19 patients. However, there is no documentation on the use of this technology in COVID-19 research, due to the absence of usable information and annotated data. Wang et al. [95], studied the respiratory tract of patients with COVID-19 and other usually cold and flu respiratory tract patients. In addition, for the diagnosis of COVID-19, they suggested a model of respiratory simulation (called BI-ATGRU). The BI-AT-GRU model involves the bidirectional bids and attention of the GRU neural network and can differentiate between 6 forms of clinical respiratory types, such as Eupnea, Tachypnea, Bradypnea, Biots, Cheyne-Stokes, and CentralApnea. Sharma et al. [80] intended to create the laboratory diagnostic techniques of COVID-19 for sputum testing. To test biomarkers in acoustics, the project , called Coswara, used cough, breath, and speech sounds. Data were collected for nine distinct vowel sounds. Some other features were also collected. The nine voices carry on varying patterns of body-breathing. From audio files, visual and temporary spectral features have been extracted. Tasks for classification and data curation are under operation.

A portable device called FluSense [21] [1] was developed by the University of Massachusetts Amherst. Figure 6 shows the components of the FluSense machine operated by an AI-based neural network that can real-time identify cough and crowd size and directly evaluate and collect data for flu-like diseases such as COVID-19. A microphone array and a thermal camera configuration and neural computing engine are used by the FluSense to actively and constantly render speech and cough signals as well as real-time adjustments in the crowd density on the edge. These sources of knowledge helps to determine the time of campaigns for vaccination for the flu, possible travel restrictions, delivery of drugs, and more [96]. FluSense developers claim that the new edge computing platform, which is believed to be used in medical centers, will extend the health monitoring instruments used to predict influenza and other viruses including the epidemics of COVID-19.

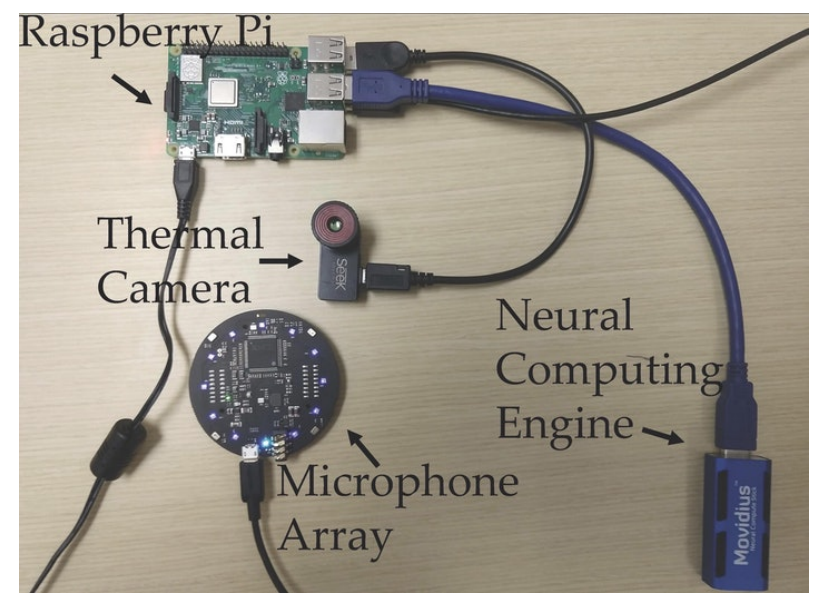

Fig. 6. The FluSense device houses these components [21] [1].

In [1], it has shown that FluSense accurately predicted the daily number of patient using a Pearson Correlation Coefficient of $95 \%$. Both respiratory diseases or additional health data were not considered by the FluSense platform. Information on cough is important and necessary, but not appropriate for all respiratory infections to be used. They gather data from a number of sources. An online website devoted to spreading cough sounds from COVID-19 patients, Ravelo [71], launched a cough AI-based system against COVID-19. 
The Bill and Melinda Gates Foundation is supportive of this initiative. The mechanism is plain. A individual downloads a sputum recording and is requested to provide information such as symptoms, other diseases, gender, and geographic location, to decide if the person is from a specific area has a COVID19 infection or not. The procedure also asks individuals for a photo of the results of their COVID-19 test. They will create an ML or DL-based algorithm once they have enough data and check if the cough sounds associated with COVID-19 infection can be accurately determined. Iqbal et al. [37] addressed an anonymous structure that applies the role of the mobile app recognition to obtain and evaluate suspicious cough sounds of individuals to assess whether a person is healthy or suffering from respiratory disease.

A cross-sectional system between vocal cords (coughing and breathing) was created by researchers at the University of Cambridge [10] to recognise safe and unhealthy individuals. Speech sounds are applied to differentiate between COVID19, normal persons, and asthma. The three binary separation functions are structured as follows:

- Separating positive users of COVID-19 from negative users.

- Separate COVID-19 cough users from healthy cough users.

- Separate COVID-19 cough users from asthma users who have declared coughing.

In the community driven data collection, more than 7,000 unique users (approximately $10 \mathrm{~K}$ samples) participated, from which more than 200 registered positive COVID-19. Typical methods of audio enhancement have been used to maximise a data set's sample size. For the classification task, three classifications were used, namely, LR, Gradient Boosting Trees (GBT), and SVM. To compare efficiency, the analysis used the combined curve area under the curve ( AUC). In all three binary split trades, more than 70 percent of AUCs are registered. In order to distinguish, the researchers used respiratory tests and found that the AUC was around $60 \%$. However, because of the high number of characteristics, the AUC progresses to around $80 \%$ per operation when coughing and respiratory inputs are merged into phases. Table III provides a description of AI-based ML and DL approaches for speech and audio analysis regarding to health problems with COVID-19.

\section{A. COVID-19 Diagnosis Based on Non-invasive Measure- ments}

Maghdid et al. [48] was planned for the invisible COVID-19 diagnostic system based on smart phone sensors. Smart phones may be used in the proposed system to gather possible patient disease features. For example, through a recording feature, sensors can detect a patient's voice and can detect the body temperature of a patient via a finger recognition function. The data collected is subsequently transferred to a cloud server funded by AI for disease diagnosis and analysis. Indeed, it takes quite a while to compare different CT pictures, and radiologists can't physically finish their comprehension. In this way, the new system allows radiologists and empowers individuals in suspicious cases to make successful and accurate decisions.

\section{ML AND DL For DRUG AND VACCINE DEVELOPMENT}

In combination with a large amount of data, the capacity for automatic abstract component learning has had a major impact on the efficacious use of ML. Drug discovery and vaccination affected two fields of high importance, where ML provided integrated property predictions, behaviour prediction, reaction prediction, and ligand-protein interactions. mDiverse drug development programs and vaccines for SARS-CoV-2 and COVID-19 have been suggested focused on proteomics and genomics studies. One of the main contributions to intelligent medicine is the use of ML and DL in the development of new medicines and vaccines and plays a major function in the battle against COVID-19.

\section{A. ML and DL For Vaccine Development}

In this context, ML and DL play two important supporting roles: dissemination of vaccine components by observing the viral protein structure, and helping medical researchers review a large number of important research papers at an incredible rate. Three main types of vaccines are available: vaccines for any pathogen, such as flu or MMR, use deadly or compromised immune system infections; subunit vaccines (e.g., pertussis, shingles) use only part of the virus, such as protein; and vaccines for nucleic acid inject viral genes into human cells to improve the response of the body [19]. The newest is the COVID-19 vaccine, which began trials in the United States this week. AI helps speed up the growth of subunits and nucleic acids.

In analyzing how it functions, understanding protein composition is critical. Researchers can produce drugs that work in various protein shapes once the situation is acknowledged. But testing any protein structure will take quite a long time before finding its unique 3D structure. The method of evaluating protein structure and its genetic sequence can be simplified by AI systems based on DL.

Google DeepMind [78] [31] introduced AlphaFold in January, an advanced and specialised system that forecasts the formation of $3 \mathrm{D}$ proteins using their genetic sequence. In the beginning of March, the system was tested in COVID19. To asses the scientific community for understanding the virus, DeepMind has published a protein prediction for several untreated proteins related to SARS-CoV-2 since it is the main cause for COVID-19. Meanwhile, scientists at the University of Texas at Austin and the National Institutes of Health have applied a standard biological method to establish the first 3D atomic map on the scale of a spike protein component of a virus that binds to human cells. Other Coronaviruses, including SARS-CoV and MERS-CoV, have spent years collaborating with the team responsible for this crucial breakthrough. Accurate predictions for this spike structure were provided by one of the predictions released by AlphaFold. Computer simulations to build $3 \mathrm{D}$ atom models on the SARS-CoV-2 protein scale neatly related to the results found on the UT Austin board 
TABLE III

AN OVERVIEW OF AI-BASED ML AND DL METHODS FOR COVID-19 SPEECH AND AUDIO ANALYSIS.

\begin{tabular}{|c|c|c|c|c|}
\hline Author & Usage & ML/DL Method & Dataset & Performance \\
\hline Miranda et al. $|52|$ & TB Diagnosis & MFB, MFCC, STFT with CNN & $\begin{array}{l}\text { Google audio dataset which includes } 1.8 \text { million } \\
\text { Youtube Freesound videos and audio database }\end{array}$ & $94.6 \%$ AUC \\
\hline Yadav et al. $|98|$ & $\begin{array}{l}\text { Asthmatic Dis- } \\
\text { covery }\end{array}$ & $\begin{array}{l}\text { INTERSPEECH } 2013 \text { (The basis } \\
\text { of the Computational Paralinguistics } \\
\text { challenge acoustic features) }\end{array}$ & $\begin{array}{l}\text { Speech from } 47 \text { asthmatic and } 48 \text { healthy con- } \\
\text { trols }\end{array}$ & $48 \%$ Accuracy \\
\hline Larson et al. [44] & $\begin{array}{l}\text { Avoid } \\
\text { recording } \\
\text { of speech while } \\
\text { hearing cough }\end{array}$ & $\begin{array}{l}\text { PCA on audio spectrograms, FFT } \\
\text { coefficients and random separation }\end{array}$ & $\begin{array}{l}\text { Acted cough from } 17 \text { patients having } 8 \text { people } \\
\text { cough due to cold weather, } 3 \text { patients because } \\
\text { of Asthama, } 1 \text { patient because of allergies and } \\
5 \text { patients who cough due to chronic cough } \\
\text { condition }\end{array}$ & $\begin{array}{l}92 \% \text { of } \\
\text { positive rue } \\
\text { and } 50 \% \text { of } \\
\text { false positive } \\
\text { rate }\end{array}$ \\
\hline Simply et al. $|83|$ & $\begin{array}{l}\text { Obstructive } \\
\text { sleep apnea } \\
\text { (OSA) } \\
\text { acquisition } \\
\text { from sounds } \\
\text { breathing in } \\
\text { speech }\end{array}$ & $\begin{array}{l}\text { MFCCs have a single layer neural } \\
\text { network for respiration and MFCCs, } \\
\text { strength, tone, kurtosis and ZCR } \\
\text { with OV SVM separation }\end{array}$ & $\begin{array}{l}90 \text { Male subjects' speech and sleep quality } \\
\text { measures using WatchPAT }\end{array}$ & $\begin{array}{l}50 \% \text { for } \\
\text { breathing } \\
\text { detection using } \\
\text { Cohen's kappa } \\
\text { coefficient and } \\
54 \% \text { using } \\
\text { OSA detection }\end{array}$ \\
\hline Routray et al. $|74|$ & $\begin{array}{l}\text { Automatic } \\
\text { speech } \\
\text { breathing } \\
\text { average rating }\end{array}$ & $\begin{array}{l}\text { Cepstrogram and support Vector ma- } \\
\text { chine with radial base work }\end{array}$ & $\begin{array}{l}16 \text { recording of speeches } 21 \text {-year-old group } \\
\text { participants age means }\end{array}$ & $\begin{array}{ll}89 \% & \text { F1- } \\
\text { measure } & \end{array}$ \\
\hline $\begin{array}{l}\text { Nallanthighal and } \\
\text { Strik [55] }\end{array}$ & $\begin{array}{l}\text { Receiving } \\
\text { a breathing } \\
\text { signal in } \\
\text { conversational } \\
\text { speech }\end{array}$ & Pectrogram with CNN and RNN & $\begin{array}{l}20 \text { healthy speeches recordings using a micro- } \\
\text { phone and a breathing signal using two respira- } \\
\text { tor transducers belts }\end{array}$ & $\begin{array}{l}91.2 \% \text { respira- } \\
\text { tory sensitivity }\end{array}$ \\
\hline Partila et al. [64] & $\begin{array}{l}\text { Pressure } \\
\text { detection in } \\
\text { speech in cases } \\
\text { such as a } \\
\text { car accident, } \\
\text { domestic } \\
\text { violence, and } \\
\text { conditions near } \\
\text { death }\end{array}$ & $\begin{array}{l}\text { LLD and functional characteristics } \\
\text { are obtained using openSMILE with } \\
\text { SVM and CNN separators }\end{array}$ & $\begin{array}{l}312 \text { emergency call recording of } 112 \text { Emer- } \\
\text { gency Cable Emergency Plan from the Czech } \\
\text { Republic }\end{array}$ & $\begin{array}{lr}87.9 \% & \text { accuracy } \\
\text { using } & \text { SVM } \\
\text { and } & 87.5 \% \\
\text { Accuracy } & \text { using } \\
\text { CNN }\end{array}$ \\
\hline
\end{tabular}

were also used by [22] at the Institute for Protein Design at the University of Washington. By constructing new proteins to minimize Coronavirus, they are currently continuing on this work. In principle, these proteins will conform to a protein spike that protects healthy cells from being invaded by viral particles.

Researchers merged AI with cloud computing to stop the Spike protein from binding to the ACE2 receptor in human cells and to produce a possible vaccine for COVID-19 [72]. Flinders University researchers studied the COVID-19 virus and then applied their data to model a vaccine called Covax19 as shown in Figure 7

In order to determine how the virus was harming human cells, the researchers utilized computer generated models of the $\mathrm{S}$ protein and its human receptor, the enzyme-converting angiotensin 2 (ACE2). Then, they attempted to produce a vaccine that could prevent this mechanism. The team used the most innovative AI and advanced cloud computing technology to speed up the production of vaccines Qitevic [90] [32].

Herst et al. [28], detected GenBank's SARS-CoV-2 protein sequence and applied MSA algorithm to specify the sequence of nucleocapsid phosphoprotein in future peptide sequencing. It also indicates that in EBOV (West African Epidemic 20132016) survivors, a peptide vaccine dependent on CD8 + T-cell immunity is appropriate and feasible. Ong et al. [59], used the methods of ML and reverse vaccinology (RV) to forecast

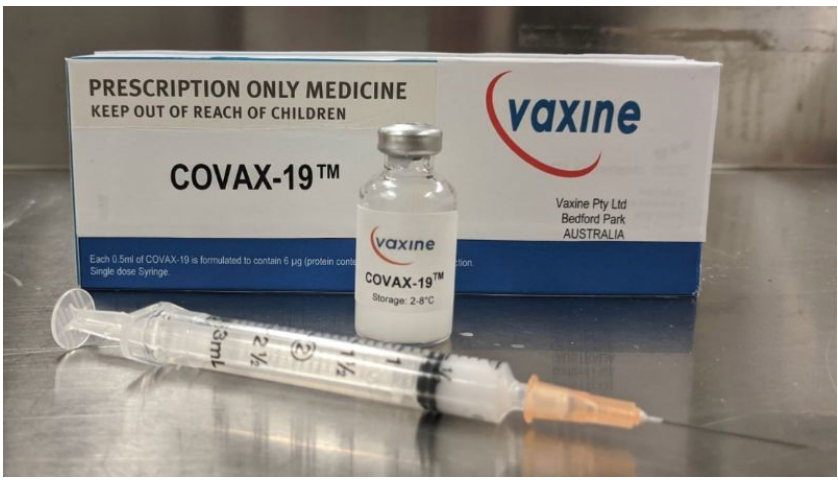

Fig. 7. Covax-19 $19^{\mathrm{TM}}$ is an Australian-designed COVID-19 vaccine modeled by AI-based technologies [85].

and test possible COVID-19 vaccines. In order to describe promising baptismal candidates, they used RV to analyze bioinformatics pathogen genomes. The SARS-CoV-2 sequence was discovered. The known proteins of the coronavirus types, including SARS-CoV, MERS-CoV, HCoV-229E, HCoVOC43, HCoV-NL63, and HCoV-HKU1, were obtained from Uniprot proteomes. They subsequently used Vaxign and Vaxign-ML to analyze and forecast the functioning of biological signals of the complete proteome of Coronaviruses. Next, using structural, vector support, proximity neighbor, random forest, 
and overgrowth (XGB) strategies, they formed the VaxignML model in the context of ML and RV and determined the level of the protein. To determine the rate of protegenicity of all WSH-CoV-2 proteins separating Wuhan-Hu-1, downloaded from NCBI, the most powerful XGB model was chosen. A good immunodeficiency vaccine is considered a protein with a protegenicity point greater than $90 \%$ (weight of F1-points $>94 \%$ in five-fold combined validation). For phylogenetic study, the NSP3 protein was chosen and the immunogenicity of NSP3 was assessed by predicting MHC-I and MHC-II T cells and epitopes of Linear B cells.

Some other researchers [18] from the Computer Science and AI Laboratory (CSAIL) of MIT have currently adopted a novel strategy in which they have applied an optimized ML-based method that choose peptides (short amino acid fibers) that are expected to give large vaccine numbers. The "OptiVax" design software incorporates ways of designing new peptide medicines, evaluating existing vaccines and increasing the composition of existing vaccines. For their ability to express themselves in response to antibodies, peptides gain mechanical learning points in this program and are chosen to expand coverage of those who can benefit from this vaccine.

Rahman et al. [69], applied immunoinformatics methods and relative methods to produce a SARS-CoV-2 anti-peptide vaccine consisting of $\mathrm{S}$ (spike), $\mathrm{E}$ (envelop), and $\mathrm{M}$ (membrane epitopes)) protein. To predict B-specific epitopes in S-proteins, they applied the Ellipro antibody epitope predictive system. In order to forecast and visualize a specific protein sequence or Bcell epitope in a structure, Ellipro uses several ML techniques. In addition, the epitope-based vaccine design of COVID-19 was studied by Sarkar et al. [75]. They applied an SVM technique to forecast the toxicity of chosen epitopes. Prachar et al [66], applied 19 joined epitope-HLA tools, including the IEDB, ANN, and PSSM algorithm, to forecast and validate 174 epitopes of SARS-CoV-2.

\section{B. Drug Development}

With the development of a predictive learning model, AI systems based on ML and DL are used in field design and perform a fast-paced test to accurately represent the performance. The AI systems can easily classify drugs that can battle infectious diseases such as COVID-19 with a drug recovery process. With this breakthrough, which is a medical evidencebased tool, will potentially enhance drug accessibility, preparation, care, and recorded patient outcomes COVID-19. Drug development is a very dangerous, lengthy and costly phase. While it takes ten to fifteen years to make a new molecular venture, the success rate is only $2.01 \%$ as reported by the Eastern Research Group (ERG) [97]. For the treatment of a never-considered medicinal indication, the idea of drug repurposing reuses old medications. It is an experimental strategy to the detection of pre-approved, discontinued, shelved and investigational drugs for the treatment of other diseases with approved restatement.The development of conventional drugs generally involves five steps [53]:

- Drug discovery and development.

- Drug pre-clinical research.
- Drug clinical research.

- FDA drug review.

- FDA drug after-market safety control and development.

But only drug repurposing requires four steps [53]:

- Compound identification.

- Compound acquisition.

- Drug clinical research.

- FDA drug after-market safety control and development.

The discovery and development of new molecular structure are slow, time consuming, and expensive. Therefore, the best option is to reconstruct the approved drugs for SARS-COV-2 therapy. In this case, in the treatment of viral infection, Chloroquine (CQ) and its hydroxyl analogue Hydroxychloroquine (HCQ) have been identified. These drugs have antimalarial efficacy and have also been shown to treat COVID-19 in vitro [42].

One AI-based drug development team focuses on the discovery at the molecular level of new drug-like compounds. Beck et al. [9], suggested a DL-based drug-target interaction model called Molecule Transformer-Drug Target Interaction (MT-DTI) to predict potential drugs for COVID-19. SMILES strings and amino-acid sequences were used in the MT-DTI model to classify target proteins with 3D crystal structures. The findings showed that atazanavir, an antiretroviral drug used to treat and prevent the human immunodeficiency virus (HIV), is the best chemical compound with an inhibitory potency with $\mathrm{Kd}$ of $94.94 \mathrm{nM}$ against 3C-like SARS-CoV2 proteinase, followed by remdesivir (113.13 nM), efavirenz (199.17 nM), ritonavir (204.05 nM), and dolutegravir (336.91 $\mathrm{nM})$. From the databases of NCBI, Drug Target Popular (DTC), and BindingDB, the authors obtained the amino acid sequences of $3 \mathrm{C}$-like proteases and associated antiviral drugs and drug targets. In addition, to determine the binding affinity between 3,410 drugs and SARS-CoV-2 3CLpro, they utilised a molecular docking and virtual screening method (AutoDock Vina). Six possible medicines, such as Remdesivir, Atazanavir, Efavirenz, Ritonavir, Dolutegravir, Kaletra (lopinavir/ritonavir), produced experimental results. Note that, in a clinical trial, Remdesivir looks promising.

Using effective machine learning techniques, two similaritybased methods, KronRLS [62] and SimBoost [26] have been proposed. But there are two downsides to this matrix. First, the representation of features is reduced, which would cause the prediction faulty. Second, it involves the estimation of the matrix of similarity, which in the training phase will limit the maximum number of molecules. A deep learning based DTI model, DeepDTA [60], was proposed to address these limitations. It is a CNN-based end-to-end model that waives the feature engineering requirement. From a raw molecule and protein sequence, the model automatically finds useful features. The performance was seen on two publicly accessible DTI benchmarks, i.e. Sim-Boost and KronRLS. To extract features representations from the raw protein sequences and SMILES strings and train them, they used CNN blocks and combined these features representations to input into a deep CNN, naming it DeepDTA. They applied Smith-Waterman (SW) and Pubchem Similarity algorithms to process the proteins 
TABLE IV

A SUMMARY OF DRUG DEVELOPMENT OF COVID-19 BASED ON AI-BASED ML AND DL METHODS [13].

\begin{tabular}{|c|c|c|c|c|}
\hline Author & Method & Role of ML/DL Method & COVID-19 Target & Potent In Drugs \\
\hline Moskal et al. [54] & $\begin{array}{lr}\text { VAE, } & \text { CNN, } \\
\text { LSTM, } & \text { and } \\
\text { MLP } & \end{array}$ & $\begin{array}{l}\text { Help in generating SMILES strings and } \\
\text { molecules }\end{array}$ & N/A & 110 Drugs \\
\hline Beck et al. $\mid 9]$ & MT-DTI & $\begin{array}{l}\text { Predict binding affinity between drugs and pro- } \\
\text { tein targets }\end{array}$ & 3CLpro, RdRp, Helicase & 6 Drugs \\
\hline Hu et al. |33| & $\mathrm{DL}$ & $\begin{array}{l}\text { Predict binding between drugs and protein tar- } \\
\text { gets }\end{array}$ & 3CLpro, RdRp & 10 drugs \\
\hline Kadioglu et al. $\mid 41\rceil$ & $\begin{array}{l}\mathrm{NN} \text { and Naive } \\
\text { Bayes }\end{array}$ & Construct drug likelihood prediction model & spike protein and more & 3 drugs \\
\hline Hofmarcher et al. $|29|$ & ChemAI & $\begin{array}{l}\text { Classify the moecules effects on COVID- } 19 \\
\text { proteases }\end{array}$ & 3CLpro, PLpro & 30,000 molecules \\
\hline Bung et al. $[11]$ & RNN and RL & Classify protease inhibitor molecules & 3CLpro & 31 compounds \\
\hline $\begin{array}{l}\text { Zhavoronkov et al. } \\
{[102]}\end{array}$ & $28 \mathrm{ML}$ & Generate new molecular structures for 3CLpro & 3CLpro & 100 molecules \\
\hline Tang et al. $|86|$ & RL and DQN & $\begin{array}{l}\text { Predict molecules and lead compounds for each } \\
\text { target fragment }\end{array}$ & 3CLpro & 47 compounds \\
\hline
\end{tabular}

and ligands' pair-wise similarities, respectively. In tarining the patterns of the data, three alternative combinations applied this knowledge as an input to the proposed ad enhanced DeepDTA model. The following are the three alternative combinations for training this model:

- Training only compound representation.

- Training only protein sequence representation.

- Training both protein representation and compound representations.

The last one is the combined model. The combined model is used in many researches [3], [9], [34] for COVID-19 drug repurposing.

They introduced a network-based DL method called deepDR [100], for in silico drug repurposing by ten integrated networks. In particular, with the support of a deep Autoencoder, the deepDR understands medication high-stage features from the heterogeneous networks. Then, with clinically recorded drug-disease pairs, the learned low-dimensional features representations of drugs is encoded and decoded together by a Variational Autoencoder to deduce applicants for licensed drugs that they were no longer initially allowed. DeepDR revealed a high performance of $90.8 \%$ AUC, surpassing other methods based on CNN and ML. Some of the other drug testing, repurposing and discovery strategies based on deep learning for COVID-19 are DeepPurpose [34], kGCN [43], DeepChem [70], and D3Targets-2019-nCoV [82].

Bung et al. [11] undertook the progress of modern SARSCoV-2 3CLpro chemical frameworks focused on deep learning technology. For protease inhibitor molecule molecules, they established an RL-based RNN model and found a tiny set that prefers chemical space. Eventually, 2515 SMILES format protease inhibitor molecules were collected from the ChEMBL database to train, in which each sequence of the SMILES was treated as a time series and as well as the location and symbol. The release of tiny molecules was embedded with minimal force into the 3CLpro structure and was determined on the basis of a visual test note acquired by the selection of participants with anti-SARS-CoV-2. Tang et al. [86] analyzed
3CLpro with a 3D structure similar to SARS-CoV and tested it as an appealing goal for the development of anti-COVID19 drugs. They developed a comprehensive Q-learning network (called ADQN-FBDD) to produce leading SARS-CoV-2 3CLpro compounds. They collected 284 molecules as SARSCoV-2 3CLpro inhibitors. These molecules are classified using an enhanced BRICS technique to gain a SARS-CoV-2 3CLpro targeted library. Thereafter, the proposed ADQN-FBDD network trains each piece of the target and classifies the corresponding molecules with the leading elements. Through the Structure-Based Optimization Policy (SBOP), 47 alternatives were identified to the inhibitory effects on SARS-CoV2 3CLpro in these compounds, which are considered to be anti-SARS-CoV-2 drugs. A summary of drug development of COVID-19 based on AI-based ML and DL methods is shown in Table IV that provides a description of COVID-19 drug production based on AI-based ML and DL methods.

\section{Challenges And Future Directions}

In this section, we provide details on the challenges using ML and DL to tackle the problem of COVID-19. Also, we show future research directions that ML and DL can contribute to the battle of COVID-19.

\section{A. Challenges}

AI-based ML and DL applications in COVID-19 research are currently facing several obstacles, such as legislation, scarcity and unavailability of large-scale training data, vast noisy data and rumors, limited awareness of the intersection between computer science and medicine, data privacy and security issue, unreliable usability of text data, and more.

- Regulation. As the pandemic increases and maximizes the regular number of confirmed infected and deceased cases, numerous steps have been considered to contain this pandemic, e.g. Lockdown and social distancing. In the time of pandemic, authorities play a key part in identifying regulations and policies that can promote the 
participation of citizens, researchers, scientists, business owners, medical centers, technology giants and major corporations to avert any barrier to COVID-19 prevention.

- scarcity and unavailability of large-scale training data. Many AI-based DL techniques depend on large-scale training data, including medical imaging and different details of the environment. However, there are inadequate datasets available for AI because of the rapid explosion of COVID-19. In reality, interpreting training samples is time-consuming and may need trained medical professionals.

- Noisy data and online rumors. The challenges emerge from depending on portable online social media; without any significant changes, large audio information and false reports about COVID-19 have been reported in different online outlets. However, in judging and filtering audio and error data, AI-based ML and DL algorithms appear to be slow. Also, with the usage of noisy data, the results of the AI-based ML and DL algorithms become biased. The usage, functionality, and the performance of AIbased methods are reduced by this issue, particularly in pandemic predictions and spread analysis.

- Lacking in the intersection of computer science and medicine fields. Most AI-based ML and DL researchers come from computer science major, but strong specialization in medical imaging, bioinformatics, virology, and many other related fields is also required to involve other medical knowledge for the use of ML and DL in the COVID-19 war. To deal with COVID-19, it is therefore important to organize the collaboration of experts from various fields and to incorporate the information of multiple studies.

- Data privacy and protection. The cost of collecting personal privacy data in the age of big data and AI is very weak. Many governments aim to collect a range of personal information including ID number, contact number, personal data, and medical data, in the face of public health issues such as COVID-19. A problem worth addressing is how to efficiently preserve your privacy and human rights throughout AI-based discovery and processing.

- Incorrect structural and unstructural data (e.g. image, text, and numerical data). Having an ambiguous and incorrect information in text descriptions can be a challenge. Huge quantities of information from different sources can be incorrect. Also, excess data makes it impossible for valuable pieces of information to be extracted.

- COVID-19 early diagnosis using medical imaging e.g. Chest X-ray and CT scan. Dealing with unbalanced datasets due to inadequate medical imaging and long training period knowledge from COVID-19 and unable to clarify the challenges of the findings.

- Screen and triage patients, find functional therapies and cures, risk assessments, survival predictions, health care and medical resource planning. The challenge is to collect physical attributes and treatment results for patients. Another difficulty is to deal with poor quality data that can lead to forecasts that are biassed and unreliable.

\section{B. Future Research Direction}

AI-based ML and DL systems can also take part to the war against COVID-19 from the next possible directions.

- Detection of non-contact disease. The use of automated image classification in X-ray and CT imaging will effectively prevent the chance of disease transmissions from patients among radiologists during COVID-19 epidemics. For patient pose, detection of X-ray and CT images, and camera facilities, AI-based ML and DL systems can be involved.

- Remote video diagnosis and consultations. It is possible to use the combination of AI and Natural Language Processing (NLP) techniques to build remote video diagnostic programs and robot systems to provide COVID-19 patient visits and first group diagnoses.

- Biological research. AI-based ML and DL systems can be used in the context of biological research to identify protein composition and viral factors via accurate biomedical knowledge analysis, such as significant protein structures, genetic sequences, and viral trajectories.

- Drug development and vaccination. AI-based ML and DL systems can not only be applied to identify possible drugs and vaccines, but can also be used to mimic drugprotein and vaccine-receptor interactions, thus predicting future drug and vaccine reactions for people with various COVID-19 patients.

- Identification and screening of fake information. To provide credible, factual, and scientific information about the COVID-19 outbreak, AI-based ML and DL systems can be applied to minimize and delete false news and audio data on internet forums.

- Impact assessment and evaluation. In order to analyse the effect of various social control modes on disease transmission, different types of simulations may use AIbased ML and DL systems. They can then be used to assess reliable and scientific approaches for diseases control and prevention in the population.

- Patient contact tracking. AI-based ML and DL system can monitor and track the characteristics of people neighboring to patients with COVID-19 by creating social networks and knowledge graphs, thereby accurately predicting and monitoring the potential spread of the disease.

- Smart robots. In programs such as sanitation in public areas, product delivery, and patient treatment without the need for human resources, intelligent robots are supposed to be used. This will stop the spreading of the virus from COVID-19.

- Future work with descriptive AI-based ML and DL techniques. The efficacy of deep learning models and graphical characteristics that lead to the differences between COVID-19 and other forms of pneumonia needs to be clarified. This will help radiologists and practitioners gain an awareness of the virus and accurately analyse possible X-ray and CT scans of the Coronavirus. 
- COVID-19 diagnosis and treatment, which one is important? Both are essential, but finding a cure for COVID-19 is definitely more essential. We find that most of the existing AI-based ML and DL strategies are more centered on identifying COVID-19 from the existing literature in this survey. In order to find the COVID-19 treatment, more future research work based on ML and $\mathrm{DL}$ is required.

\section{CONCLUSION}

The COVID-19 outbreak has had a profound effect on the wellbeing of people world wide and the number of disease-related deaths continues to grow globally. Although technology has penetrated our daily lives with great success, especially in ML and DL, AI has also contributed to supporting people in the difficult battle against COVID-19. We explored the AI-based ML and DL methods for COVID-19 diagnosis and treatment in this survey. In addition, in the war against COVID-19, we summarized the AI-based ML and DL methods and the available datasets, tools, and performance. This survey offers a detailed overview of the existing state-of-the-art methodologies and applications for ML and DL researchers and the wider health community with descriptions of how ML and DL and data will boost the status of COVID-19, and more studies to avoid the outbreak of COVID-19. Challenges and potential guidance were also presented using ML and DL.

\section{ACKNOWLEDGMENT}

This work is funded by Research and Development Grants Program for National Research Institutions and Centers (GRANTS), Target Research Program, Infectious Disease Research Grant Program, King Abdulaziz City for Science and Technology (KACST), Kingdom of Saudi Arabia, grant number (5-20-01-007-0001).

\section{REFERENCES}

[1] Forsad Al Hossain, Andrew A Lover, George A Corey, Nicholas G Reich, and Tauhidur Rahman. Flusense: a contactless syndromic surveillance platform for influenza-like illness in hospital waiting areas. Proceedings of the ACM on Interactive, Mobile, Wearable and Ubiquitous Technologies, 4(1):1-28, 2020.

[2] Health IT Analytics. Deep Learning Models Can Detect COVID-19 in Chest CT Scans. https://healthitanalytics.com/news/deep-learning\begin{tabular}{|l|l|l}
\hline models-can-detect-covid-19-in-chest-ct-scans. 2020. [Online; ac- &
\end{tabular} cessed 3-September-2020].

[3] Muhammad Umer Anwar, Farjad Adnan, Asma Abro, Muhammad Rayyan Khan, Asad Ur Rehman, Muhammad Osama, Saad Javed, Ahmadullah Baig, Muhammad Raffey Shabbir, Muhammad Zaman Assir, et al. Combined deep learning and molecular docking simulations approach identifies potentially effective fda approved drugs for repurposing against sars-cov-2, 2020.

[4] Ioannis D Apostolopoulos, Sokratis I Aznaouridis, and Mpesiana A Tzani. Extracting possibly representative covid-19 biomarkers from $\mathrm{x}$-ray images with deep learning approach and image data related to pulmonary diseases. Journal of Medical and Biological Engineering, page $1,2020$.

[5] Ioannis D Apostolopoulos and Tzani A Mpesiana. Covid-19: automatic detection from x-ray images utilizing transfer learning with convolutional neural networks. Physical and Engineering Sciences in Medicine, page 1, 2020.

[6] Xiang Bai, Cong Fang, Yu Zhou, Song Bai, Zaiyi Liu, Liming Xia, Qianlan Chen, Yongchao Xu, Tian Xia, Shi Gong, et al. Predicting covid-19 malignant progression with ai techniques. 2020.
[7] Sanhita Basu and Sushmita Mitra. Deep learning for screening covid19 using chest X-ray images. arXiv preprint arXiv:2004.10507, 2020.

[8] BBVA. Artificial intelligence to detect COVID-19 in less than a second using x-rays. https://www.bbva.com/en/artificial-intelligence-to-detectcovid-19-in-less-than-a-second-using- $x$-rays/2019. [Online; accessed 6-September-2020].

[9] Bo Ram Beck, Bonggun Shin, Yoonjung Choi, Sungsoo Park, and Keunsoo Kang. Predicting commercially available antiviral drugs that may act on the novel coronavirus (sars-cov-2) through a drugtarget interaction deep learning model. Computational and structural biotechnology journal, 2020.

[10] Chloë Brown, Jagmohan Chauhan, Andreas Grammenos, Jing Han, Apinan Hasthanasombat, Dimitris Spathis, Tong Xia, Pietro Cicuta, and Cecilia Mascolo. Exploring automatic diagnosis of covid-19 from crowdsourced respiratory sound data. arXiv preprint arXiv:2006.05919, 2020.

[11] Navneet Bung, Sowmya Ramaswamy Krishnan, Gopalakrishnan Bulusu, and Arijit Roy. De novo design of new chemical entities (nces) for sars-cov-2 using artificial intelligence. ChemRxiv, 2020.

[12] Charmaine Butt, Jagpal Gill, David Chun, and Benson A Babu. Deep learning system to screen coronavirus disease 2019 pneumonia. Applied Intelligence, page 1, 2020.

[13] Jianguo Chen, Kenli Li, Zhaolei Zhang, Keqin Li, and Philip S Yu. A survey on applications of artificial intelligence in fighting against covid-19. arXiv preprint arXiv:2007.02202, 2020.

[14] Jun Chen, Lianlian Wu, Jun Zhang, Liang Zhang, Dexin Gong, Yilin Zhao, Shan Hu, Yonggui Wang, Xiao Hu, Biqing Zheng, et al. Deep learning-based model for detecting 2019 novel coronavirus pneumonia on high-resolution computed tomography: a prospective study. MedRxiv, 2020.

[15] Muhammad EH Chowdhury, Tawsifur Rahman, Amith Khandakar, Rashid Mazhar, Muhammad Abdul Kadir, Zaid Bin Mahbub, Khandaker Reajul Islam, Muhammad Salman Khan, Atif Iqbal, Nasser AlEmadi, et al. Can ai help in screening viral and covid-19 pneumonia? arXiv preprint arXiv:2003.13145, 2020

[16] Joseph Paul Cohen, Lan Dao, Paul Morrison, Karsten Roth, Yoshua Bengio, Beiyi Shen, Almas Abbasi, Mahsa Hoshmand-Kochi, Marzyeh Ghassemi, Haifang Li, et al. Predicting covid-19 pneumonia severity on chest X-ray with deep learning. arXiv preprint arXiv:2005.11856, 2020.

[17] Dipayan Das, KC Santosh, and Umapada Pal. Truncated inception net: Covid-19 outbreak screening using chest $\mathrm{x}$-rays. Physical and engineering sciences in medicine, pages 1-11, 2020.

[18] Srishti Deoras. How ML Is Assisting In Development Of Covid19 Vaccines. https://analyticsindiamag.com/how-ml-is-assisting-indevelopment-of-covid-19-vaccines/] 2020. [Online; accessed 19September-2020].

[19] Oren Etzioni and Nicole Decario. AI Can Help Scientists Find a Covid-19 Vaccine. https://www.wired.com/story/opinion-ai-can-helpfind-scientists-find-a-covid-19-vaccine/ 2020. [Online; accessed 16September-2020].

[20] HEALTH EUROPA. Using Artificial Intelligence to determine COVID19 severity. https://www.healtheuropa.eu/using-artificial-intelligenceto-determine-covid-19-severity/100501//2020. [Online; accessed 12September-2020].

[21] FluSense. AI-Powered COVID-19 Cough Analyzer App Assesses Respiratory Health and Associated Risks. https://www.umass.edu/ gateway/feature/flusense 2020. [Online; accessed 16-September-2020].

[22] Institute for Protein Design. Rosetta's role in fighting coronavirus. https://www.ipd.uw.edu/2020/02/rosettas-role-in-fightingcoronavirus/, 2020. [Online; accessed 10-September-2020].

[23] Biraja Ghoshal and Allan Tucker. Estimating uncertainty and interpretability in deep learning for coronavirus (covid-19) detection. arXiv preprint arXiv:2003.10769, 2020.

[24] Ophir Gozes, Maayan Frid-Adar, Hayit Greenspan, Patrick D Browning, Huangqi Zhang, Wenbin Ji, Adam Bernheim, and Eliot Siegel Rapid ai development cycle for the coronavirus (covid-19) pandemic: Initial results for automated detection \& patient monitoring using deep learning ct image analysis. arXiv preprint arXiv:2003.05037, 2020.

[25] Lawrence O Hall, Rahul Paul, Dmitry B Goldgof, and Gregory M Goldgof. Finding covid-19 from chest x-rays using deep learning on a small dataset. arXiv preprint arXiv:2004.02060, 2020.

[26] Tong He, Marten Heidemeyer, Fuqiang Ban, Artem Cherkasov, and Martin Ester. Simboost: a read-across approach for predicting drugtarget binding affinities using gradient boosting machines. Journal of cheminformatics, 9(1):1-14, 2017. 
[27] Ezz El-Din Hemdan, Marwa A Shouman, and Mohamed Esmail Karar. Covidx-net: A framework of deep learning classifiers to diagnose covid-19 in X-ray images. arXiv preprint arXiv:2003.11055, 2020.

[28] Charles V Herst, Scott Burkholz, John Sidney, Alessandro Sette, Paul E Harris, Shane Massey, Trevor Brasel, Edecio Cunha-Neto, Daniela S Rosa, William Chong Hang Chao, et al. An effective ctl peptide vaccine for ebola zaire based on survivors' cd8+ targeting of a particular nucleocapsid protein epitope with potential implications for covid-19 vaccine design. Vaccine, 2020.

[29] Markus Hofmarcher, Andreas Mayr, Elisabeth Rumetshofer, Peter Ruch, Philipp Renz, Johannes Schimunek, Philipp Seidl, Andreu Vall, Michael Widrich, Sepp Hochreiter, et al. Large-scale ligand-based virtual screening for sars-cov-2 inhibitors using deep neural networks. Available at SSRN 3561442, 2020.

[30] HospiMedica. AI-Powered COVID-19 Cough Analyzer App Assesses Respiratory Health and Associated Risks. https://www.hospimedica.com/covid-19/articles/294784217/aipowered-covid-19-cough-analyzer-app-assesses-respiratory-healthand-associated-risks.html 2020. [Online; accessed 14-September2020].

[31] HospiMedica. Scientists Use Cloud-Based Supercomputing and AI to Develop COVID-19 Treatments and Vaccine Models. https://www.hospimedica.com/covid-19/articles/294784537/scientistsuse-cloud-based-supercomputing-and-ai-to-develop-covid-19treatments-and-vaccine-models.html] $2020 . \quad$ [Online; accessed 15-September-2020].

[32] HospiMedica. Scientists Use Cloud-Based Supercomputing and AI to Develop COVID-19 Treatments and Vaccine Models. https://www.hospimedica.com/covid-19/articles/294784537/scientistsuse-cloud-based-supercomputing-and-ai-to-develop-covid-19treatments-and-vaccine-models.html] $2020 . \quad$ [Online; accessed 18-September-2020].

[33] Fan Hu, Jiaxin Jiang, and Peng Yin. Prediction of potential commercially inhibitors against sars-cov-2 by multi-task deep model. arXiv preprint arXiv:2003.00728, 2020.

[34] Kexin Huang, Tianfan Fu, Lucas Glass, Marinka Zitnik, Cao Xiao, and Jimeng Sun. Deeppurpose: a deep learning library for drug-target interaction prediction and applications to repurposing and screening, 2020

[35] Lu Huang, Rui Han, Tao Ai, Pengxin Yu, Han Kang, Qian Tao, and Liming Xia. Serial quantitative chest ct assessment of covid-19: Deeplearning approach. Radiology: Cardiothoracic Imaging, 2(2):e200075, 2020.

[36] Ali Imran, Iryna Posokhova, Haneya N Qureshi, Usama Masood, Sajid Riaz, Kamran Ali, Charles N John, and Muhammad Nabeel. Ai4covid19: Ai enabled preliminary diagnosis for covid-19 from cough samples via an app. arXiv preprint arXiv:2004.01275, 2020.

[37] Mohammad Zafar Iqbal and Md Faiz Iqbal Faiz. Active surveillance for covid-19 through artificial in-telligence using concept of real-time speech-recognition mobile application to analyse cough sound. 2020.

[38] itn. CT Provides Best Diagnosis for Novel Coronavirus (COVID19). https://www.itnonline.com/content/ct-provides-best-diagnosisnovel-coronavirus-covid-19 2020. [Online; accessed 20-September2020].

[39] Cheng Jin, Weixiang Chen, Yukun Cao, Zhanwei Xu, Xin Zhang, Lei Deng, Chuansheng Zheng, Jie Zhou, Heshui Shi, and Jianjiang Feng. Development and evaluation of an ai system for covid-19 diagnosis. medRxiv, 2020.

[40] Shuo Jin, Bo Wang, Haibo Xu, Chuan Luo, Lai Wei, Wei Zhao, Xuexue Hou, Wenshuo Ma, Zhengqing Xu, Zhuozhao Zheng, et al. Ai-assisted ct imaging analysis for covid-19 screening: Building and deploying a medical ai system in four weeks. medRxiv, 2020.

[41] Onat Kadioglu, Mohamed Saeed, H Johannes Greten, and Thomas Efferth. Identification of novel compounds against three targets of sars cov- 2 coronavirus by combined virtual screening and supervised machine learning. Bull. World Health Organ, 2020.

[42] Mohammad Sultan Khuroo, Ahmad A Sofi, and Mohammad Khuroo. Chloroquine and hydroxychloroquine in coronavirus disease 2019 (covid-19). facts, fiction \& the hype. a critical appraisal. International journal of antimicrobial agents, page 106101, 2020.

[43] Ryosuke Kojima, Shoichi Ishida, Masateru Ohta, Hiroaki Iwata, Teruki Honma, and Yasushi Okuno. kgcn: a graph-based deep learning framework for chemical structures. Journal of Cheminformatics, 12:1$10,2020$.

[44] Eric C Larson, TienJui Lee, Sean Liu, Margaret Rosenfeld, and Shwetak N Patel. Accurate and privacy preserving cough sensing using a low-cost microphone. In Proceedings of the 13th international conference on Ubiquitous computing, pages 375-384, 2011.

[45] Lin Li, Lixin Qin, Zeguo Xu, Youbing Yin, Xin Wang, Bin Kong, Junjie Bai, Yi Lu, Zhenghan Fang, Qi Song, et al. Artificial intelligence distinguishes covid-19 from community acquired pneumonia on chest ct. Radiology, 2020.

[46] Mohamed Loey, Florentin Smarandache, and Nour Eldeen M Khalifa. Within the lack of chest covid-19 x-ray dataset: A novel detection model based on gan and deep transfer learning. Symmetry, 12(4):651, 2020.

[47] Halgurd S Maghdid, Aras T Asaad, Kayhan Zrar Ghafoor, Ali Safaa Sadiq, and Muhammad Khurram Khan. Diagnosing covid-19 pneumonia from $\mathrm{x}$-ray and ct images using deep learning and transfer learning algorithms. arXiv preprint arXiv:2004.00038, 2020.

[48] Halgurd S Maghdid, Kayhan Zrar Ghafoor, Ali Safaa Sadiq, Kevin Curran, and Khaled Rabie. A novel ai-enabled framework to diagnose coronavirus covid 19 using smartphone embedded sensors: Design study. arXiv preprint arXiv:2003.07434, 2020.

[49] Arpan Mangal, Surya Kalia, Harish Rajgopal, Krithika Rangarajan, Vinay Namboodiri, Subhashis Banerjee, and Chetan Arora. Covidaid: Covid-19 detection using chest X-ray. arXiv preprint arXiv:2004.09803, 2020.

[50] Xueyan Mei, Hao-Chih Lee, Kai-yue Diao, Mingqian Huang, Bin Lin, Chenyu Liu, Zongyu Xie, Yixuan Ma, Philip M Robson, Michael Chung, et al. Artificial intelligence-enabled rapid diagnosis of patients with covid-19. Nature Medicine, pages 1-5, 2020.

[51] Shervin Minaee, Rahele Kafieh, Milan Sonka, Shakib Yazdani, and Ghazaleh Jamalipour Soufi. Deep-covid: Predicting covid-19 from chest $\mathrm{x}$-ray images using deep transfer learning. arXiv preprint arXiv:2004.09363, 2020.

[52] Igor DS Miranda, Andreas H Diacon, and Thomas R Niesler. A comparative study of features for acoustic cough detection using deep architectures. In 2019 41st Annual International Conference of the IEEE Engineering in Medicine and Biology Society (EMBC), pages 2601-2605. IEEE, 2019.

[53] Sweta Mohanty, Md Harun Ai Rashid, Mayank Mridul, Chandana Mohanty, and Swati Swayamsiddha. Application of artificial intelligence in covid-19 drug repurposing. Diabetes \& Metabolic Syndrome: Clinical Research \& Reviews, 2020.

[54] Martyna Moskal, Wiktor Beker, Rafał Roszak, Ewa P Gajewska, Agnieszka Wołos, Karol Molga, Sara Szymkuć, and Bartosz A Grzybowski. Suggestions for second-pass anti-covid-19 drugs based on the artificial intelligence measures of molecular similarity, shape and pharmacophore distribution. 2020.

[55] Venkata Srikanth Nallanthighal and H Strik. Deep sensing of breathing signal during conversational speech. 2019.

[56] Ali Narin, Ceren Kaya, and Ziynet Pamuk. Automatic detection of coronavirus disease (covid-19) using x-ray images and deep convolutional neural networks. arXiv preprint arXiv:2003.10849, 2020.

[57] Qianqian Ni, Zhi Yuan Sun, Li Qi, Wen Chen, Yi Yang, Li Wang, Xinyuan Zhang, Liu Yang, Yi Fang, Zijian Xing, et al. A deep learning approach to characterize 2019 coronavirus disease (covid-19) pneumonia in chest ct images. European radiology, pages 1-11, 2020.

[58] European Society of Medical Imaging Informatics. Automated diagnosis and quantitative analysis of COVID-19 on imaging. https: //imagingcovid19ai.eu/ 2020. [Online; accessed 1-September-2020].

[59] Edison Ong, Mei U Wong, Anthony Huffman, and Yongqun He. Covid19 coronavirus vaccine design using reverse vaccinology and machine learning. BioRxiv, 2020.

[60] Hakime Öztürk, Arzucan Özgür, and Elif Ozkirimli. Deepdta: deep drug-target binding affinity prediction. Bioinformatics, 34(17):i821i829, 2018.

[61] Tulin Ozturk, Muhammed Talo, Eylul Azra Yildirim, Ulas Baran Baloglu, Ozal Yildirim, and U Rajendra Acharya. Automated detection of covid-19 cases using deep neural networks with x-ray images. Computers in Biology and Medicine, page 103792, 2020.

[62] Tapio Pahikkala, Antti Airola, Sami Pietilä, Sushil Shakyawar, Agnieszka Szwajda, Jing Tang, and Tero Aittokallio. Toward more realistic drug-target interaction predictions. Briefings in bioinformatics, 16(2):325-337, 2015.

[63] Mohammad Khalid Pandit and Shoaib Amin Banday. Sars n-cov219 detection from chest x-ray images using deep neural networks. International Journal of Pervasive Computing and Communications, 2020.

[64] Pavol Partila, Jaromir Tovarek, Jan Rozhon, and Jakub Jalowiczor. Human stress detection from the speech in danger situation. In Mobile Multimedia/Image Processing, Security, and Applications 2019, volume 
10993, page 109930U. International Society for Optics and Photonics, 2019.

[65] PhysicsWorld. AI checks CT scans for COVID-19. https:// physicsworld.com/a/ai-checks-ct-scans-for-covid-19/ 2020. [Online; accessed 1-September-2020].

[66] Marek Prachar, Sune Justesen, Daniel B Steen-Jensen, Stephan P Thorgrimsen, Erik Jurgons, Ole Winther, and Frederik Otzen Bagger. Covid-19 vaccine candidates: Prediction and validation of 174 sarscov-2 epitopes. bioRxiv, 2020.

[67] Xiaolong Qi, Zicheng Jiang, Qian Yu, Chuxiao Shao, Hongguang Zhang, Hongmei Yue, Baoyi Ma, Yuancheng Wang, Chuan Liu, Xiangpan Meng, et al. Machine learning-based ct radiomics model for predicting hospital stay in patients with pneumonia associated with sars-cov-2 infection: A multicenter study. medRxiv, 2020.

[68] RadBoudumc. Evaluation of an AI system for detection of COVID-19 on Chest X-Ray images. https://www.radboudumc.nl/en/news/2020/ evaluation-of-an-ai-system-for-detection-of-covid-19-on-chest- $x$-rayimages 2020. [Online; accessed 13-September-2020].

[69] M Shaminur Rahman, M Nazmul Hoque, M Rafiul Islam, Salma Akter, ASM Rubayet-Ul-Alam, Mohammad Anwar Siddique, Otun Saha, Md Mizanur Rahaman, Munawar Sultana, Keith A Crandall, et al. Epitope-based chimeric peptide vaccine design against $\mathrm{s}, \mathrm{m}$ and e proteins of sars-cov-2 etiologic agent of global pandemic covid-19: an in silico approach. PeerJ, 8:e9572, 2020.

[70] Bharath Ramsundar, Peter Eastman, Patrick Walters, and Vijay Pande. Deep learning for the life sciences: applying deep learning to genomics, microscopy, drug discovery, and more. " O'Reilly Media, Inc.", 2019.

[71] Jenny Ravelo. This nonprofit needs your cough sounds to detect COVID-1. https://www.devex.com/news/this-nonprofit-needs-yourcough-sounds-to-detect-covid-19-97141 2020. [Online; accessed 12September-2020].

[72] Victoria Rees. AI and Cloud Computing Used to Develop COVID19 Vaccine. https://www.drugtargetreview.com/news/59650/ai-andcloud-computing-used-to-develop-covid-19-vaccine/] 2020. [Online; accessed 17-September-2020].

[73] Erik Ridley. AI can assess COVID-19 severity on chest $x$ rays. https://www.auntminnie.com/index.aspx?sec=ser\&sub=def\&pag= dis\&ItemID=129674 2020. [Online; accessed 12-September-2020].

[74] Aurobinda Routray et al. Automatic measurement of speech breathing rate. In 2019 27th European Signal Processing Conference (EUSIPCO), pages 1-5. IEEE, 2019.

[75] Bishajit Sarkar, Md Asad Ullah, Fatema Tuz Johora, Masuma Afrin Taniya, and Yusha Araf. The essential facts of wuhan novel coronavirus outbreak in china and epitope-based vaccine designing against 2019ncov. BioRxiv, 2020.

[76] Björn W Schuller, Dagmar M Schuller, Kun Qian, Juan Liu, Huaiyuan Zheng, and Xiao Li. Covid-19 and computer audition: An overview on what speech \& sound analysis could contribute in the sars-cov-2 corona crisis. arXiv preprint arXiv:2003.11117, 2020.

[77] Megan Scudellari. Hospitals Deploy AI Tools to Detect COVID-19 on Chest Scans. https://spectrum.ieee.org/the-human-os/biomedical/ imaging/hospitals-deploy-ai-tools-detect-covid19-chest-scans 2020. [Online; accessed 10-September-2020].

[78] Anderw Senior, John Jumper, Demis Hassabis, and Pushmeet Kohli. Alphafold: Using ai for scientific discovery. DeepMind. Recuperado de: https://deepmind. com/blog/alphafold, 2018.

[79] Prabira Kumar Sethy and Santi Kumari Behera. Detection of coronavirus disease (covid-19) based on deep features. Preprints, 2020030300:2020, 2020.

[80] Neeraj Sharma, Prashant Krishnan, Rohit Kumar, Shreyas Ramoji, Srikanth Raj Chetupalli, Prasanta Kumar Ghosh, Sriram Ganapathy, et al. Coswara-a database of breathing, cough, and voice sounds for covid-19 diagnosis. arXiv preprint arXiv:2005.10548, 2020.

[81] Feng Shi, Liming Xia, Fei Shan, Dijia Wu, Ying Wei, Huan Yuan, Huiting Jiang, Yaozong Gao, He Sui, and Dinggang Shen. Largescale screening of covid-19 from community acquired pneumonia using infection size-aware classification. arXiv preprint arXiv:2003.09860, 2020.

[82] Yulong Shi, Xinben Zhang, Kaijie Mu, Cheng Peng, Zhengdan Zhu, Xiaoyu Wang, Yanqing Yang, Zhijian Xu, and Weiliang Zhu. D3targets2019-ncov: a webserver for predicting drug targets and for multitarget and multi-site based virtual screening against covid-19. Acta Pharmaceutica Sinica B, 2020.

[83] Ruby M Simply, Eliran Dafna, and Yaniv Zigel. Obstructive sleep apnea (osa) classification using analysis of breathing sounds during speech. In 2018 26th European Signal Processing Conference (EUSIPCO), pages 1132-1136. IEEE, 2018.
[84] Mohammed A Soghaier, Khwaja MI Saeed, and Khushhal K Zaman. Public health emergency of international concern (pheic) has declared twice in 2014; polio and ebola at the top. AIMS public health, 2(2):218, 2015.

[85] TABIP. Coronavirus Vaccine Candidate Developed In Adelaide Lab To Start Human Trials. https://covid19.tabipacademy.com/2020/07/ 03/coronavirus-vaccine-candidate-developed-in-adelaide-lab-to-starthuman-trials/ 2020. [Online; accessed 20-September-2020].

[86] Bowen Tang, Fengming He, Dongpeng Liu, Meijuan Fang, Zhen Wu, and Dong Xu. Ai-aided design of novel targeted covalent inhibitors against sars-cov-2. bioRxiv, 2020.

[87] Cranfield University. Artificial intelligence spots COVID-19 in chest X-rays. https://pharmaphorum.com/news/artificialintelligence-spotscovid-19-from-chest-X-rays/, 2020. [Online; accessed 12-September2020].

[88] Cranfield University. Using artificial intelligence to detect COVID19 in X-rays. https://medicalxpress.com/news/2020-05-artificialintelligence-covid-X-rays.html 2020. [Online; accessed 11-September2020].

[89] Cranfield University. Using machine learning to detect COVID19 in X-rays. https://tectales.com/ai/using-ai-to-detect-covid-19-in-Xrays.html 2020. [Online; accessed 11-September-2020].

[90] Flinders University. Microsoft's AI for Health Supports COVID19 Vaccine. https://medicalxpress.com/news/2020-08-microsoft-aihealth-covid-vaccine.html 2020. [Online; accessed 18-September2020].

[91] New York University. App determines COVID-19 disease severity using artificial intelligence, biomarkers. https://www.sciencedaily.com/ releases/2020/06/200603132529.htm 2020. [Online; accessed 14September-2020].

[92] VIDA. LungPrint Clinical Solutions. https://vidalung.ai/clinical_ solutions/ 2020. [Online; accessed 2-September-2020].

[93] Linda Wang and Alexander Wong. Covid-net: A tailored deep convolutional neural network design for detection of covid-19 cases from chest X-ray images. arXiv preprint arXiv:2003.09871, 2020.

[94] Shuai Wang, Bo Kang, Jinlu Ma, Xianjun Zeng, Mingming Xiao, Jia Guo, Mengjiao Cai, Jingyi Yang, Yaodong Li, Xiangfei Meng, et al A deep learning algorithm using ct images to screen for corona virus disease (covid-19). MedRxiv, 2020.

[95] Yunlu Wang, Menghan Hu, Qingli Li, Xiao-Ping Zhang, Guangtao Zhai, and Nan Yao. Abnormal respiratory patterns classifier may contribute to large-scale screening of people infected with covid-19 in an accurate and unobtrusive manner. arXiv preprint arXiv:2002.05534, 2020.

[96] ET Health World. Scientists develop portable AI device that can use coughing sounds to monitor COVID-19 trends. https://health.economictimes.indiatimes.com/news/diagnostics/ scientists-develop-portable-ai-device-that-can-use-coughing-sounds\begin{tabular}{lll}
\hline to-monitor-covid-19-trends/74730537 & $2020 . \quad$ [Online; accessed
\end{tabular} 11-September-2020].

[97] Hanqing Xue, Jie Li, Haozhe Xie, and Yadong Wang. Review of drug repositioning approaches and resources. International journal of biological sciences, 14(10):1232, 2018.

[98] Shivani Yadav, Merugu Keerthana, Dipanjan Gope, Prasanta Kumar Ghosh, et al. Analysis of acoustic features for speech sound based classification of asthmatic and healthy subjects. In ICASSP 20202020 IEEE International Conference on Acoustics, Speech and Signal Processing (ICASSP), pages 6789-6793. IEEE, 2020.

[99] Seung Hoon Yoo, Hui Geng, Tin Lok Chiu, Siu Ki Yu, Dae Chul Cho, Jin Heo, Min Sung Choi, Il Hyun Choi, Cong Cung Van, Nguen Viet Nhung, et al. Deep learning-based decision-tree classifier for covid19 diagnosis from chest $\mathrm{x}$-ray imaging. Frontiers in medicine, 7:427, 2020.

[100] Xiangxiang Zeng, Siyi Zhu, Xiangrong Liu, Yadi Zhou, Ruth Nussinov, and Feixiong Cheng. deepdr: a network-based deep learning approach to in silico drug repositioning. Bioinformatics, 35(24):5191-5198, 2019.

[101] Kang Zhang, Xiaohong Liu, Jun Shen, Zhihuan Li, Ye Sang, Xingwang Wu, Yunfei Zha, Wenhua Liang, Chengdi Wang, Ke Wang, et al. Clinically applicable ai system for accurate diagnosis, quantitative measurements, and prognosis of covid-19 pneumonia using computed tomography. Cell, 2020.

[102] Alex Zhavoronkov, Vladimir Aladinskiy, Alexander Zhebrak, Bogdan Zagribelnyy, Victor Terentiev, Dmitry S Bezrukov, Daniil Polykovskiy, Rim Shayakhmetov, Andrey Filimonov, Philipp Orekhov, et al. Potential covid-2019 3c-like protease inhibitors designed using generative 
deep learning approaches. Insilico Medicine Hong Kong Ltd A, 307:E1, 2020.

[103] Chuansheng Zheng, Xianbo Deng, Qing Fu, Qiang Zhou, Jiapei Feng, Hui Ma, Wenyu Liu, and Xinggang Wang. Deep learning-based detection for covid-19 from chest ct using weak label. medRxiv, 2020. 